\title{
ON THE INVERSE PROBLEM OF THE DISCRETE CALCULUS OF VARIATIONS
}

\author{
GIORGIO GUBBIOTTI
}

\begin{abstract}
In this paper we present an algorithm to find the discrete Lagrangian for an autonomous recurrence relation of arbitrary even order $2 k$ with $k>1$. The method is based on the existence of a set of differential operators called annihilation operators which can be used to convert a functional equation into a system of linear partial differential equations. This completely solves the inverse problem of the calculus of variations in this setting.
\end{abstract}

\section{INTRODUCTION}

One of the most powerful tools in Mathematical Physics since the times of Euler and Lagrange is the calculus of variations. The variational formulation of mechanics where the equations of motion arise as the minimum of an action functional (the so-called Hamilton's principle), is fundamental in the developing of theoretical mechanics and its foundations are present in each textbook on this subject [20,36, 58]. Beside this, the application of calculus of variations goes beyond mechanics as many important mathematical problems, e.g. the isoperimetrical problem and the catenary, can be formulated in terms of calculus of variations. For a more complete outlook on the calculus of variations, its scopes and its applications we refer to standard textbooks on the subject [10, 15, 19, 32, 50].

An important problem regarding the calculus of variations is to determine when a system of differential equations are the Euler-Lagrange equations for some variational problem. This problem is called the inverse problem of the calculus of variations and has a long and interesting history. It was was first addressed by Jacobi in the case of second-order scalar ordinary differential equations [27-30]. In this case it turns out that the answer is that such kind of equations admit infinitely many inequivalent Lagrangians. Jacobi's proof use the so-called Jacobi Last Multiplier, which can be explicitly used to construct Lagrangians. This proof can be found in [58]. The same result was found also by different authors after Jacobi, usually with no explicit mention of his work, see [11,24]. The general case of this problem remains unsolved, whereas several important results for particular cases where presented during the XX century. In particular we mention that, under certain restrictions, the general case can be addressed by a method developed by the Italian mathematician V. Volterra [57]. Such method can be understood in a modern formalism through the theory of variational complexes and homotopy [54]. Volterra's result is therefore now known as the homotopy formula. A complete solution in the case of the systems of two second-order ordinary differential equations was given by Douglas in 1941 [12]. It is worth to note that Douglas' solution

Date: May 20, 2019.

2010 Mathematics Subject Classification. 70S05; 39A99. 
have been interpreted in terms of geometrical calculus in [8,51] using the important advances on the subject given in [2]. In the scalar case necessary and sufficient conditions on the existence of a Lagrangian are known up to eight-order equations. Indeed in [13] such conditions were derived in the case of fourth-order equations, whereas in [33] a set of conditions for the existence of a Lagrangian for sixth- and eight-order equations was given. For systems of $n$ second-order differential equations some results on radially symmetric systems exist [7, 25]. For a complete, yet accessible historical introduction on the subject we refer to [48, Chap. 4]. For a more updated exposition on the results obtained on the subject in the last 30 years we refer to the reviews [52,56] and references therein.

We mention that the relation of the Jacobi Last Multiplier with the inverse problem of the calculus of variations was often neglected in literature. The Jacobi Last Multiplier itself was rediscovered several times by researchers unaware of its properties. For an historical perspective on this subject we refer to [41,43, 47] and references therein. Moreover, we observe that the Jacobi Last Multiplier can be used to find Lagrangians for differential equations of order higher than two [42] and for non-dissipative systems of second-order equations [44,49].

In this paper we give some condition on the existence of a Lagrangian in the discrete scalar setting. To be more precise, we will reduce the condition of existence of a Lagrangian for a scalar difference equation of arbitrary even order $2 k$, with $k>1$ to the solution of an overdetermined system of linear partial differential equations. The fact that the system is linear and overdetermined, means that in general it possible to solve it without an excessive effort. Our solution to the problem is constructive: solving such system one can either find the Lagrangian or conclude that it does not exist. This result pave the way to various applications, for instance in the classification of difference equations based on their variational structure, or their integrability properties. This approach has been already applied to understand how integrability arises in a family of fourth-order maps with two given invariants 21,22.

The inverse problem of discrete calculus of variations was considered from the point of view of variational complexes and homotopy in [26]. In such paper a result analogous to the homotopy formula for the continuous case was proved. We mention that an early description of the difference variational complex appeared in [35].

The plan of the paper is following: in Section 2 we introduce the basis of the discrete calculus of variations for scalar difference equations. We give a self-contained account of all the fundamental results we are going to use based on the original papers on the subject $[4,40,53,55$. In Section 3 we present our main result which is given by Theorem 3.4. This theorem, along with its Corollary 3.5, allows to prove or disprove the existence of a Lagrangian in our setting. Section 4 is then devoted to examples. We present several examples from the recent literature, even of equations of arbitrarily high order. Finally in Section 5 we give some conclusions and outlook. In particular we discuss the possibility of using the method presented in section 3 in the framework of the geometric integration theory [5, 6, 34, and we present a comparison with the homotopy approach given in [26].

\section{Discrete LAGRANGIANS AND THEIR BASIC PROPERTIES}

In this section we introduce discrete Lagrangians and those of their properties we are going to use throughout the paper. Some of these properties were discussed 
already in [40. Later accounts on these properties of discrete Lagrangians can be found in [4,55] and more recently in [53. We refer to these papers and references therein for a more complete overview on the subject.

Throughout this paper we are going to consider consider recurrence relation of even order $2 k$, equivalently called a scalar difference equation, i.e. functional equations of the following from:

$$
x_{n+k}=F\left(x_{n+k-1}, x_{n+k-2}, \ldots, x_{n-k}\right) .
$$

where $k \geq 1$. To be a well-posed equation of order $2 k$ we impose the non-degeneracy condition:

$$
\frac{\partial F}{\partial x_{n-k}} \neq 0 \text {. }
$$

Throughout the paper we will always consider this condition to be satisfied.

A discrete action of order $k$ is a linear functional of the form:

$$
S\left[x_{n}\right]=\sum_{n \in \mathbb{Z}} L\left(x_{n+k}, x_{n+k-1}, \ldots, x_{n}\right) .
$$

The summand function

$$
L=L\left(x_{n+k}, x_{n+k-1}, \ldots, x_{n}\right)
$$

is called a discrete Lagrangian.

Usually we think difference equation (2.1) and discrete Lagrangians (2.4) to be autonomous, i.e. not explicitly dependent on $n$. For this reason in the general fomulæ (2.1) and (2.4) we omitted the index $n$ in the hand side. However, we underline that all the reasoning presented in this paper also work in the non-autonomous case, with the appropriate care. In particular we will underline with appropriate remarks when a result can be simplified in the autonomous setting. On the other side, when a result requires to be be discussed in the non-autonomous setting we will always place the subscript $n$ and explain why it is needed.

From the variation of the discrete action (2.3) we obtain that the stationary points of the discrete action satisfy the following difference equation [40]:

$$
\sum_{l=0}^{k} T_{n}^{-l} \frac{\partial L}{\partial x_{n+l}}\left(x_{n+k}, x_{n+k-1}, \ldots, x_{n}\right)=0 .
$$

In formula (2.5) $T_{n}$ is the translation operator acting on any function $f_{n}=f_{n}\left(x_{n+l}, \ldots, x_{n+m}\right)$ depending on a finite number of shifts of $x_{n}$ and possibly on the independent variable $n$ as:

$$
T_{n} f_{n}\left(x_{n+l}, \ldots, x_{n+m}\right)=f_{n+1}\left(x_{n+l+1}, \ldots, x_{n+m+1}\right) .
$$

Equation (2.5) is called the discrete Euler-Lagrange equation corresponding to the discrete Lagrangian $L$ (2.4). When no ambiguity is possible, we will simply address equation (2.5) as the discrete Euler-Lagrange equation. The left hand side of the discrete Euler-Lagrange equations (2.5) is sometimes called the variational derivative of the action (2.3) and denoted by $\delta S / \delta x_{n}$.

We say that a discrete Lagrangian (2.4) is a discrete Lagrangian for the difference equation (2.1) if its discrete Euler-Lagrange equations (2.5) coincide with (2.1).

Remark 2.1. The expression of the Euler-Lagrange equation given in equation (2.5) is valid both for autonomous and non-autonomous Lagrangians. In particular in 
the case of autonomous Lagrangians $L=L\left(x_{n+k}, \ldots, x_{n}\right)$ its expression can be simplified to:

$$
\sum_{l=0}^{k} \frac{\partial L}{\partial x_{n}}\left(x_{n+k-l}, x_{n+k-1}, \ldots, x_{n-l}\right)=0 .
$$

On the other hand if the Lagrangian depends explicitly on $n, L=L_{n}$ we can write formula (2.5) as:

$$
\sum_{l=0}^{k} \frac{\partial L_{n-l}}{\partial x_{n}}\left(x_{n+k-l}, x_{n+k-1}, \ldots, x_{n-l}\right)=0 .
$$

When developing the theory of discrete Lagrangians it is good to consider both expressions (2.5) and (2.8) as the former is more abstract and useful for theoretical purposes, while the latter is more useful in explicit computations.

In particular formula (2.5) allow us to think of the variational derivative as a linear operator acting on functions of the form (2.4). That is, we can state the following definition:

Definition 2.1. The linear differential-difference operator:

$$
\mathcal{E}=\sum_{l=0}^{k} T_{n}^{-l} \frac{\partial}{\partial x_{n+l}}
$$

is called the Euler operator.

The Euler-Lagrange equation is written in terms of the Euler operator (2.9) as $\mathcal{E}(L)=0$. The following important result about the kernel of the Euler operator (2.9) holds:

Lemma 2.1. The kernel of the Euler operator (2.9) is the space of total differences, i.e. of functions $g_{n}=g_{n}\left(x_{n+k}, \ldots, x_{n}\right)$ such that there exists a function $f_{n}=$ $f_{n}\left(x_{n+k-1}, \ldots, x_{n}\right)$ such that:

$$
g_{n}\left(x_{n+k}, \ldots, x_{n}\right)=\left(T_{n}-\mathrm{Id}\right) f_{n}\left(x_{n+k-1}, \ldots, x_{n}\right),
$$

that is, the image of the operator $T_{n}-\mathrm{Id}$. Symbolically we write:

$$
\operatorname{ker} \mathcal{E}=\operatorname{Im}\left(T_{n}-\mathrm{Id}\right) .
$$

A proof of Lemma 2.1 in the context of difference variational complex can be found in 26 35]. Here we present a simple proof which does not require any knowledge of the geometrical theory of discrete calculus of variations. However, is worth showing such proof since the computational techniques employed will be used when constructing discrete Lagrangians in section 4 .

Proof. First we prove the inclusion $\operatorname{Im}\left(T_{n}-\mathrm{Id}\right) \subset \operatorname{ker} \mathcal{E}$. Assume $g_{n} \in \operatorname{Im}\left(T_{n}-\mathrm{Id}\right)$ and apply $\mathcal{E}$ to it:

$$
\mathcal{E}\left[g_{n}\left(x_{n+k}, \ldots, x_{n}\right)\right]=\mathcal{E}\left[\left(T_{n}-\mathrm{Id}\right) f_{n}\left(x_{n+k-1}, \ldots, x_{n}\right)\right] .
$$


After some trivial algebra using the definition of the Euler operator (2.9) we find following identity:

$\mathcal{E}\left[g_{n}\left(x_{n+k}, \ldots, x_{n}\right)\right]=\frac{\partial}{\partial x_{n}} \sum_{l=0}^{k}\left[f_{n+1-l}\left(x_{n+k-l}, \ldots, x_{n-l+1}\right)-f_{n-l}\left(x_{n+k-l-1}, \ldots, x_{n-l}\right)\right]$.

The sum on the right hand side of equation (2.13) is telescopic, therefore its value is given by:

$$
\mathcal{E}\left[g_{n}\left(x_{n+k}, \ldots, x_{n}\right)\right]=\frac{\partial}{\partial x_{n}}\left[f_{n+1}\left(x_{n+k}, \ldots, x_{n+1}\right)-f_{n-l}\left(x_{n-1}, \ldots, x_{n-k}\right)\right]=0 .
$$

This proves that $g_{n} \in \operatorname{ker} \mathcal{E}$.

Now, we prove the reverse inclusion $\operatorname{ker} \mathcal{E} \subset \operatorname{Im}\left(T_{n}-\mathrm{Id}\right)$. We proceed by induction on the number of points.

Case $k=1$. Assume $g_{n}=g_{n}\left(x_{n+1}, x_{n}\right) \in \operatorname{ker} \mathcal{E}$, that is:

$$
\frac{\partial g_{n}}{\partial x_{n}}\left(x_{n+1}, x_{n}\right)+\frac{\partial g_{n-1}}{\partial x_{n}}\left(x_{n}, x_{n-1}\right)=0 .
$$

Differentiating the previous equation with respect to $x_{n+1}$ we obtain that $g_{n}$ solves the following partial differential equation for all $n$ :

$$
\frac{\partial^{2} g_{n}}{\partial x_{n+1} \partial x_{n}}\left(x_{n+1}, x_{n}\right)=0 \text {. }
$$

This last equation imply:

$$
g_{n}\left(x_{n+1}, x_{n}\right)=g_{n}^{(1)}\left(x_{n+1}\right)+g_{n}^{(2)}\left(x_{n}\right) .
$$

Taking advantage of the arbitrariness of $g_{n}^{(1)}$ and $g_{n}^{(2)}$ we can write $g_{n}^{(2)}\left(x_{n+1}\right)=$ $G_{n}\left(x_{n}\right)-g_{n}^{(1)}\left(x_{n}\right)$, that is:

$$
g_{n}\left(x_{n+1}, x_{n}\right)=G_{n}\left(x_{n}\right)+\left(T_{n}-\mathrm{Id}\right) g_{n}^{(1)}\left(x_{n}\right) .
$$

As $\left(T_{n}-\mathrm{Id}\right) g_{n}^{(1)}\left(x_{n}\right) \in \operatorname{Im}\left(T_{n}-\mathrm{Id}\right)$ substituting (2.18) into (2.15) we have $G_{n}^{\prime}\left(x_{n}\right)=$ 0 . This implies $G_{n}\left(x_{n}\right)=G_{n}$, or in (2.18):

$$
g_{n}\left(x_{n+1}, x_{n}\right)=G_{n}+\left(T_{n}-\mathrm{Id}\right) g_{n}^{(1)}\left(x_{n}\right)=\left(T_{n}-\mathrm{Id}\right)\left[g_{n}^{(1)}\left(x_{n}\right)+\widetilde{G}_{n}\right] .
$$

In formula (2.19) we represented $G_{n}$ as a discrete integration $G_{n}=\left(T_{n}-\right.$ Id) $\widetilde{G}_{n}$, as we can always do when there is no explicit dependence on $x_{n}$ and its shifts. From (2.19) we have that for $k=1 g_{n} \in \operatorname{Im}\left(T_{n}-\mathrm{Id}\right)$ with

$$
f_{n}\left(x_{n}\right)=g_{n}^{(1)}\left(x_{n}\right)+\widetilde{G}_{n} .
$$

Case $k>1$. Assume $g_{n}=g_{n}\left(x_{n+k}, \ldots, x_{n}\right) \in \operatorname{ker} \mathcal{E}$ for $k>1$, and that the property $\operatorname{ker} \mathcal{E} \subset \operatorname{Im}\left(T_{n}-\mathrm{Id}\right)$ holds for all function $h_{n}$ depending on at most $k-1$ points. As $g_{n} \in \operatorname{ker} \mathcal{E}$ we have:

$$
\sum_{l=0}^{k} \frac{\partial g_{n-l}}{\partial x_{n}}\left(x_{n+k-l}, \ldots, x_{n-l}\right)=0
$$


If we differentiate (2.21) with respect to $x_{n+k}$ we obtain:

$$
\frac{\partial^{2} g_{n-l}}{\partial x_{n+k} \partial x_{n}}\left(x_{n+k-l}, \ldots, x_{n-l}\right) .
$$

Reasoning in analogous way as in the case $k=1$ we have that we can write the solution of the previous partial differential equation as:

$$
g_{n}\left(x_{n+k}, \ldots, x_{n}\right)=G_{n}\left(x_{n+k-1}, \ldots, x_{n}\right)+\left(T_{n}-\mathrm{Id}\right) \hat{g}_{n}\left(x_{n+k-1}, \ldots, x_{n}\right) .
$$

From the fact that $\operatorname{Im}\left(T_{n}-\mathrm{Id}\right) \subset \operatorname{ker} \mathcal{E}$ inserting (2.23) into (2.22) we obtain:

$$
\sum_{l=0}^{k-1} \frac{\partial G_{n-l}}{\partial x_{n}}\left(x_{n+k-l}, \ldots, x_{n-l}\right)=0 .
$$

That is, $G_{n}\left(x_{n+k-1}, \ldots, x_{n}\right) \in \operatorname{ker} \mathcal{E}$. From induction it follows:

$$
G_{n}\left(x_{n+k-1}, \ldots, x_{n}\right)=\left(T_{n}-\mathrm{Id}\right) \widetilde{g}_{n}\left(x_{n+k-2}, \ldots, x_{n}\right) .
$$

Inserting (2.25) into (2.23) we have that $g_{n}\left(x_{n+k}, \ldots, x_{n}\right) \in \operatorname{Im}\left(T_{n}-\mathrm{Id}\right)$ with the function $f_{n}\left(x_{n+k-1}, \ldots, x_{n}\right)$ given by:

$$
f_{n}\left(x_{n+k-1}, \ldots, x_{n}\right)=\widetilde{g}_{n}\left(x_{n+k-2}, \ldots, x_{n}\right)+\hat{g}_{n}\left(x_{n+k-1}, \ldots, x_{n}\right) .
$$

This concludes the proof of the lemma.

Remark 2.2. We remark that in Lemma2.1 the presence of the subscript $n$ is necessary when proving the inclusion $\operatorname{ker} \mathcal{E} \subset \operatorname{Im}\left(T_{n}-\mathrm{Id}\right)$ with the proposed technique. Indeed, if the functions $g_{n}$ and $f_{n}$ are autonomous we have an obstruction to the proof already in the case $k=1$. In this case the solution of equation (2.16) read as:

$$
g\left(x_{n+1}, x_{n}\right)=G\left(x_{n}\right)+\left(T_{n}-\mathrm{Id}\right) g_{n}^{(1)}\left(x_{n}\right) .
$$

In (2.15) this implies $G^{\prime}\left(x_{n}\right)=0$, that is $G\left(x_{n}\right)=K_{1}$, with $K_{1}$ a constant. The function:

$$
g\left(x_{n+1}, x_{n}\right)=K_{1}+\left(T_{n}-\mathrm{Id}\right) g_{n}^{(1)}\left(x_{n}\right),
$$

is in total difference form summing back equation (2.28):

$$
f_{n}\left(x_{n}\right)=g_{n}^{(1)}\left(x_{n}\right)+\left(T_{n}-\mathrm{Id}\right)^{-1}\left(K_{1}\right)+K_{2}=g_{n}^{(1)}\left(x_{n}\right)+K_{1} n+K_{2} .
$$

That is, the function $f_{n}$ will necessary depend explicitly on $n$.

Lemma 2.1 has the following immediate corollary:

Corollary 2.2. If two discrete Lagrangians $L_{1}$ and $L_{2}$ differ by a total difference, i.e. there exists a function $f_{n}=f_{n}\left(x_{n+k-1}, \ldots, x_{n}\right)$ such that:

$$
L_{2}=L_{1}+\left(T_{n}-\mathrm{Id}\right) f_{n}\left(x_{n+k-1}, \ldots, x_{n}\right),
$$

then they define the same discrete Euler-Lagrange equations.

Proof. The thesis follows immediately applying the Euler operator (2.9) to equation (2.30) and noting that the total difference on the right hand side disappears using Lemma 2.1

Using corollary (2.2) we define the following relation on discrete Lagrangians: 
Definition 2.2. Two discrete Lagrangians $L_{1}$ and $L_{2}$ are called equivalent, denoted by $\stackrel{\text { t.d. }}{=}$, if they differ for a total difference, i.e.:

$$
L_{1} \stackrel{\text { t.d. }}{=} L_{2} \Longleftrightarrow L_{1}=L_{2}+\left(T_{n}-\mathrm{Id}\right) f\left(x_{n+k-1}, \ldots, x_{n}\right) .
$$

Is it easy to prove the following proposition:

Proposition 2.3. The relation $\stackrel{t . d .}{=}$ is an equivalence relation. That is, it possesses the following properties:

Reflexivity: $L \stackrel{\text { t.d. }}{=} L$.

Symmetry: If $L_{1} \stackrel{\text { t.d. }}{=} L_{2}$ then $L_{2} \stackrel{\text { t.d. }}{=} L_{1}$.

Transitivity: If $L_{1} \stackrel{\text { t.d. }}{\equiv} L_{2}$ and $L_{2} \stackrel{\text { t.d. }}{\equiv} L_{3}$ then $L_{1} \stackrel{\text { t.d. }}{\equiv} L_{3}$.

The proof of Proposition 2.3 is trivial and it is therefore omitted.

Remark 2.3. Proposition 2.3 implies that Lagrangians are not functions, but rather equivalence classes of functions up to the equivalence relation defined by $\stackrel{\text { t.d. }}{\equiv}(2.31)$. A Lagrangian equivalent to a constant function is said to be a trivial, as its EulerLagrange equations are identically satisfied, i.e. they are not difference equations.

Finally we give the following definition:

Definition 2.3. A discrete Lagrangian $L=L\left(x_{n+k}, \ldots, x_{n}\right)$ is called normal if

$$
\frac{\partial^{2} L}{\partial x_{n+k} \partial x_{n}}\left(x_{n+k}, \ldots, x_{n}\right) \neq 0 .
$$

The importance of considering normal Lagrangians come from the fact that non-normal Lagrangians define discrete Euler-Lagrange equations of order $2 k-2$ at most. This is the content of the following lemma:

Lemma 2.4. A non-normal discrete Lagrangian $L$ defined on $k$ points is equivalent to a normal discrete Lagrangian $\widetilde{L}$ defined on $k-m$ points, where $m \in\{1, \ldots, k\}$ is the smallest integer such that:

$$
\frac{\partial^{2} L}{\partial x_{n+k-m} \partial x_{n}}\left(x_{n+k}, \ldots, x_{n}\right) \neq 0 .
$$

Proof. Assume that the discrete Lagrangian $L=L\left(x_{n+k}, \ldots, x_{n}\right)$ is non-normal. Then from definition 2.3 we have:

$$
\frac{\partial^{2} L}{\partial x_{n+k} \partial x_{n}}\left(x_{n+k}, \ldots, x_{n}\right)=0 .
$$

Solving this partial differential equation and using the same reasoning as in the proof of Lemma 2.1 we obtain that the Lagrangian $L$ has the following form:

$$
\begin{aligned}
& L=L_{1}\left(x_{n+k-1}, \ldots, x_{n}\right)+\left(T_{n}-\mathrm{Id}\right) f_{n}\left(x_{n+k-1}, \ldots, x_{n}\right) \\
& \stackrel{\mathrm{t.d} .}{=} L_{1}\left(x_{n+k-1}, \ldots, x_{n}\right) .
\end{aligned}
$$


Therefore the original discrete Lagrangian $L$ is equivalent to a discrete Lagrangian $L_{1}$ defined on $k-1$ point. The Euler-Lagrange equation of $L_{1}$ are defined on $2 k-2$ at most.

Now if:

$$
\frac{\partial^{2} L}{\partial x_{n+k-1} \partial x_{n}}=0
$$

we have that:

$$
\frac{\partial^{2} L_{1}}{\partial x_{n+k-1} \partial x_{n}}=0
$$

and the above reasoning can be iterated. Therefore, we obtain that

$$
L \stackrel{\text { t.d. }}{\equiv} L_{1}\left(x_{n+k-1}, \ldots, x_{n}\right) \stackrel{\text { t.d. }}{=} L_{2}\left(x_{n+k-2}, \ldots, x_{n}\right) .
$$

This procedure can be iterated until we reach $m \in\{1, \ldots, k\}$ defined by the condition (2.33). Defining $\widetilde{L} \equiv L_{m}$ the chain of equivalence gives us:

$$
L\left(x_{n+k}, \ldots, x_{n}\right) \stackrel{\text { t.d. }}{=} \widetilde{L}\left(x_{n+k-m}, \ldots, x_{n}\right) .
$$

This concludes the proof of the lemma.

Corollary 2.5. A discrete Lagrangian L (2.4) such that:

$$
\frac{\partial^{2} L}{\partial x_{n} \partial x_{n+m}}=0, \quad \forall m \in\{1, \ldots, k\}
$$

is trivial.

Proof. From Lemma 2.4 we have $L \stackrel{\text { t.d. }}{=} \widetilde{L}\left(x_{n}\right)$, but $\widetilde{L}\left(x_{n}\right) \stackrel{\text { t.d. }}{\equiv} \widetilde{L}_{0}$ where $\widetilde{L}_{0}$ is a constant. Therefore $L \stackrel{\text { t.d. }}{=} \widetilde{L}_{0}$ and it is a trivial Lagrangian.

Since in this paper we are interested in the inverse problem of the discrete calculus of variations for equations of the form (2.1) depending on exactly $2 k$ points, from now on, we will always consider to deal with normal discrete Lagrangians.

In the next section we see which kind of condition must be satisfied to guarantee the existence of a discrete Lagrangian for a given difference equation of order $2 k$ with $k>1$.

\section{METHOD FOR FINDING DiscRETE LAGRANGIANS}

As we said in the Introduction, we want to solve the inverse problem of the discrete calculus of variations for scalar difference equations, i.e. we want to be able to state when the difference equation (2.1) can be derived from a Lagrangian (2.4). To this end our first step is to state and prove following technical lemma:

Lemma 3.1. Consider a differential operator $\mathbf{A}$ acting on the independent variables $x_{n+k-1}, x_{n+k-2}, \ldots, x_{n-k}$, i.e.:

$$
\mathbf{A}=\sum_{i=-k}^{k-1} A_{i} \frac{\partial}{\partial x_{n+i}}, \quad A_{i}=A_{i}\left(x_{n+k-1}, \ldots, x_{n-k}\right), i=1 \ldots, k,
$$

such that for every function $G=G\left(x_{n+k}, x_{n+k-1}, \ldots, x_{n}\right)$ with $k>1$ evaluated on the solutions of the difference equation (2.1)

$$
G=G\left(F\left(x_{n+k-1}, x_{n+k-1}, \ldots, x_{n-k}\right), x_{n+k-1}, \ldots, x_{n}\right) .
$$


we have $\mathbf{A}(G) \equiv 0$ identically. Then $\mathbf{A}$ is a linear combination of the following $k-1$ differential operators:

$$
\mathbf{A}_{m}^{+}=\frac{\partial F}{\partial x_{n-m}} \frac{\partial}{\partial x_{n-k}}-\frac{\partial F}{\partial x_{n-k}} \frac{\partial}{\partial x_{n-m}}, \quad m=1, \ldots, k-1
$$

Proof. Let us consider the operator in (3.1) with unspecified coefficients $A_{i}$. We will fix the form of these coefficients by imposing the condition that the operator (3.1) applied to any function $G$ as in (3.2) is identically zero. Applying the operator (3.1) applied to a function $G$ as in (3.2) and using the chain rule we obtain:

$$
\mathbf{A}(G)=\left[\sum_{i=1}^{k} A_{-i} \frac{\partial F}{\partial x_{n-i}}\right] \frac{\partial G}{\partial F}+\sum_{i=0}^{k-1} A_{i}\left[\frac{\partial F}{\partial x_{n+i}} \frac{\partial G}{\partial F}+\frac{\partial G}{\partial x_{n+i}}\right] .
$$

Let us assume for the moment that the partial derivatives of $G$

$$
\frac{\partial G}{\partial F}, \frac{\partial G}{\partial x_{n+k-1}}, \ldots, \frac{\partial G}{\partial x_{n-k}}
$$

are functionally independent, which is the most general case. Then as the condition A $(G) \equiv 0$ in (3.4) must hold for all $G$ we can annihilate the coefficients of the partial derivatives of $G$ (3.5). From (3.4) this implies that $A_{i}=0$ for $i=0, \ldots, k$. On the other hand from the derivative with respect to the first argument we obtain the following condition:

$$
\sum_{i=1}^{k} A_{-i} \frac{\partial F}{\partial x_{n-i}}=0
$$

Equation (3.6) defines an hyperplane of dimension $k-1$ orthogonal to the gradient vector of $F$ with respect to the variables $\mathbf{x}^{-}=\left(x_{n-1}, \ldots, x_{n-k}\right)$ :

$$
\nabla_{\mathbf{x}^{-}} F=\left(\frac{\partial F}{\partial x_{n-1}}, \ldots, \frac{\partial F}{\partial x_{n-k}}\right)^{T} .
$$

A basis for the orthogonal space $\left(\nabla_{\mathbf{x}^{-}} F\right)^{\perp}$ is given by the vectors:

$$
\mathbf{v}_{1}=\left(\begin{array}{c}
\frac{\partial F}{\partial x_{n-k}} \\
0 \\
0 \\
\vdots \\
0 \\
-\frac{\partial F}{\partial x_{n-1}}
\end{array}\right), \mathbf{v}_{2}=\left(\begin{array}{c}
0 \\
\frac{\partial F}{\partial x_{n-k}} \\
0 \\
\vdots \\
0 \\
-\frac{\partial F}{\partial x_{n-2}}
\end{array}\right), \ldots, \mathbf{v}_{k-1}=\left(\begin{array}{c}
0 \\
0 \\
\vdots \\
0 \\
\frac{\partial F}{\partial x_{n-k}} \\
-\frac{\partial F}{\partial x_{n-k+1}}
\end{array}\right) .
$$

Inserting the components of this base into (3.1) we obtain the vector fields (3.3). Since (3.8) is a basis the thesis follows in the case the when the partial derivatives of $G$ (3.5) are functionally independent.

In the particular case when the partial derivatives of $G$ (3.5) are functionally dependent there exists a functional relation of the following kind:

$$
Q\left(\frac{\partial G}{\partial F}, \frac{\partial G}{\partial x_{n+k-1}}, \ldots, \frac{\partial G}{\partial x_{n}}\right)=0,
$$


with $Q$ a given function. Relation (3.9) means that $G$ solves a first-order partial differential equation. This amounts to say that the function $G$ has the form:

$$
G=G\left(I_{1}, \ldots, I_{k}\right), \quad I_{j}=I_{j}\left(F, x_{n+k-1}, \ldots, x_{n}\right),
$$

where the derivatives $\partial G / \partial I_{j}$ and functions $I_{j}$ are functionally independent and their partial derivatives are functionally independent. To each function $I_{j}$ in (3.10) the result holds because of the first part of the proof. By direct computation using the chain rule we obtain that the result holds for $G$ itself. Therefore we obtain that the result holds even in the case when the partial derivatives of $G$ (3.5) are functionally dependent. This concludes the proof of the lemma.

Remark 3.1. In the proof of Lemma 3.1 only derivatives are involved. For this reason Lemma 3.1 generalises immediately to the case when the function $F$ in (2.1) and $G$ in (3.2) depend explicitly on $n$. Therefore, to obtain the non-autonomous version of Lemma 3.1 just replace each occurrence of $F$ with $F_{n}$ and of $G$ with $G_{n}$.

Remark 3.2. We underline that in order to prove Lemma 3.1 it is fundamental to assume that the differential operator A (3.1) annihilates identically on all the functions of the form (3.2). Indeed, it is always possible to find non-trivial differential operators of the form (3.1) which annihilates a particular function, even though its first derivatives are functionally independent. A simple example of this fact arises in dimension two and it is given by radial functions. That is, the function $G(x, y)=g\left(x^{2}+y^{2}\right)$ is annihilated by the first-order linear differential operator:

$$
\mathbf{A}=y \frac{\partial}{\partial x}-x \frac{\partial}{\partial y} .
$$

However, as is it known from the theory of first-order linear partial differential equations [3], the general solution of $\mathbf{A}(G)=0$ is given by radial functions. Therefore for every non-radial function $H=H(x, y)$ we will have $\mathbf{A}(H) \neq 0$. Therefore, the linear differential operator (3.11) does not satisfy the hypotheses of Lemma 3.1.

If the equation (2.1) can be solved uniquely for $x_{n-k}$ the evolution of the difference equation is defined in both sides and we can write:

$$
x_{n-k}=\widetilde{F}\left(x_{n+k}, x_{n+k-1}, \ldots, x_{n-k+1}\right) .
$$

In this case we have a "mirrored" version of Lemma (3.1) expressed as following:

Lemma 3.2. Consider a differential operator $\widetilde{\mathbf{A}}$ acting on the independent variables $x_{n+k}, x_{n+k-2}, \ldots, x_{n-k+1}$, i.e.:

$$
\widetilde{\mathbf{A}}=\sum_{i=-k+1}^{k} \widetilde{A}_{i} \frac{\partial}{\partial x_{n+i}}, \quad \widetilde{A}_{i}=\widetilde{A}_{i}\left(x_{n+k}, \ldots, x_{n-k+1}\right), i=1 \ldots, k,
$$

such that for every function $\widetilde{G}=\widetilde{G}\left(x_{n}, x_{n-1}, \ldots, x_{n-k}\right)$ where $k>1$, evaluated on the solutions of the difference equation (3.12)

$$
\widetilde{G}=\widetilde{G}\left(x_{n}, x_{n-1}, \ldots, \widetilde{F}\left(x_{n+k}, x_{n+k-1}, \ldots, x_{n-k+1}\right)\right)
$$


we have $\widetilde{\mathbf{A}}(\widetilde{G}) \equiv 0$. Then $\widetilde{\mathbf{A}}$ is a linear combination of the following $k-1$ differential operators:

$$
\mathbf{A}_{m}^{-}=\frac{\partial \widetilde{F}}{\partial x_{n+m}} \frac{\partial}{\partial x_{n+k}}-\frac{\partial \widetilde{F}}{\partial x_{n+k}} \frac{\partial}{\partial x_{n+m}}, \quad m=1, \ldots, k-1 .
$$

Proof. Analogous to the proof of Lemma 3.1.

Remark 3.3. Analogously as Lemma 3.1 Lemma 3.2 generalises immediately to the case when the function $\widetilde{F}$ in (3.12) and $\widetilde{G}$ in (3.14) depend explicitly on $n$. To obtain the non-autonomous version of Lemma 3.2 just replace each occurrence of $\widetilde{F}$ with $\widetilde{F}_{n}$ and of $\widetilde{G}$ with $\widetilde{G}_{n}$.

Moreover, as for Lemma 3.1, Lemma 3.2 relies on the fact that the differential operator $\widetilde{\mathbf{A}}$ in (3.13) annihilates identically on all the functions of the form (3.14). It is not possible to release such hypothesis, as the same counterexample presented in Remark 3.2 is valid.

Then we state the following definition:

Definition 3.1. The differential operators introduced (3.3) and (3.15) are called annihilation operators. In particular the operators (3.3) are the forward annihilation operators, while the operators (3.15) are the backward annihilation operators.

Remark 3.4. The annihilation operators defined by equation (3.3) and (3.15) are the one-dimensional analogous of the operators $Y^{l}$ and $Z^{-l}$, for $l \in \mathbb{Z}$, defined in [16, 17, 39]. These operators have application also in the theory of Darboux integrable partial difference equations [1. In [17, 18, 23] they were used to find the first integrals of some classes of partial difference equations. These operators annihilates all the dependent shifts of a quad equation, while $\mathbf{A}_{m}^{ \pm}$annihilates the dependent shifts of a scalar difference equation.

Before going on it is important to note that the condition $k>1$ in Lemmas 3.1 and 3.2 cannot be relaxed. In fact we can prove the following, complementary result:

Lemma 3.3. Consider a first-order linear differential operator of the form

$$
\mathbf{A}=\alpha\left(x_{n}, x_{n-1}\right) \frac{\partial}{\partial x_{n}}+\beta\left(x_{n}, x_{n-1}\right) \frac{\partial}{\partial x_{n-1}},
$$

such that for every function $g=g\left(x_{n+1}, x_{n}\right)$ where $x_{n+1}$ solves a scalar secondorder difference equation of the form $x_{n+1}=f\left(x_{n}, x_{n-1}\right)$, i.e.:

$$
g=g\left(f\left(x_{n}, x_{n-1}\right), x_{n}\right) .
$$

we have $\mathbf{A}(g) \equiv 0$ identically. Then the linear differential operator $\mathbf{A}$ is trivial, i.e. $\mathbf{A} \equiv 0$.

Analogously, consider a first-order differential operator of the form

$$
\widetilde{\mathbf{A}}=\widetilde{\alpha}\left(x_{n+1}, x_{n}\right) \frac{\partial}{\partial x_{n+1}}+\widetilde{\beta}\left(x_{n+1}, x_{n}\right) \frac{\partial}{\partial x_{n}}
$$

such that for every function $\widetilde{g}=\widetilde{g}\left(x_{n}, x_{n-1}\right)$ where $x_{n-1}$ solves a scalar secondorder difference equation of the form $x_{n-1}=\widetilde{f}\left(x_{n+1}, x_{n}\right)$, i.e.:

$$
\widetilde{g}=\widetilde{g}\left(x_{n}, \widetilde{f}\left(x_{n+1}, x_{n}\right)\right) .
$$


we have $\widetilde{\mathbf{A}}(\widetilde{g}) \equiv 0$. Then the linear differential operator $\widetilde{\mathbf{A}} \equiv 0$ is trivial, i.e. $\widetilde{\mathbf{A}}$.

Proof. Applying the operator (3.16) to the function $g$ we obtain using the chain rule:

$$
\begin{aligned}
\mathbf{A}(g) & =\alpha \frac{\partial g}{\partial x_{n}}\left(f\left(x_{n}, x_{n-1}\right), x_{n}\right)+\beta \frac{\partial g}{\partial x_{n-1}}\left(f\left(x_{n}, x_{n-1}\right), x_{n}\right) \\
& =\left[\alpha \frac{\partial f}{\partial x_{n}}+\beta \frac{\partial f}{\partial x_{n-1}}\right] \frac{\partial g}{\partial f}+\alpha \frac{\partial g}{\partial x_{n}} .
\end{aligned}
$$

Assuming that the derivatives of $g$ with respect to its arguments are independent from the arbitrariness of $g$ we must annihilate their coefficients. This implies that $\alpha=\beta \equiv 0$. When the derivatives are no longer independent we can use the same argument as in Lemma 3.1 to reduce to the case when they are independent.

Performing the same reasoning in the in case when $\widetilde{g}$ is given by (3.19) and the general first-order differential operator $\widetilde{\mathbf{A}}(3.18)$ we obtain $\widetilde{\alpha}=\widetilde{\beta} \equiv 0$ and the proof is completed.

Using the annihilation operators $\mathbf{A}_{m}^{ \pm}$we can prove our main result. As this result relies on the general expression for the Euler-Lagrange equation (2.5), we will formulate it in the non-autonomous case, when such formula can be expressed explicit as equation (2.8).

Theorem 3.4. Assume that there exists a discrete Lagrangian $L=L_{n}$ (2.4) for equation (2.1), where $k>1$ and the right-hand side can depend explicitly on n, i.e. $F=F_{n}$. Then the Lagrangian $L_{n}$ satisfies the following system of linear partial differential equations:

$$
\frac{\partial}{\partial x_{n-k}}\left\{\left(\frac{\partial F_{n}}{\partial x_{n-k}}\right)^{-1} \mathbf{A}_{m}^{+}\left[\frac{\partial L_{n-k}}{\partial x_{n}}\left(x_{n}, \ldots, x_{n-k}\right)\right]\right\}=0,
$$

where $m=1, \ldots, k-1$.

Moreover, if the equation (2.1) can be solved uniquely for $x_{n-k}$ and the evolution in the backward direction is given by equation (3.12) where the right-hand side can depend explicitly on $n$, i.e. $\widetilde{F}=\widetilde{F}_{n}$ then the discrete Lagrangian $L_{n}$ (2.4) satisfies the following system of linear partial differential equations:

$$
\frac{\partial}{\partial x_{n+k}}\left\{\left(\frac{\partial \widetilde{F}_{n}}{\partial x_{n+k}}\right)^{-1} \mathbf{A}_{m}^{-}\left[\frac{\partial L_{n}}{\partial x_{n}}\left(x_{n+k}, \ldots, x_{n}\right)\right]\right\}=0
$$

where $m=1, \ldots, k-1$.

Proof. Applying the annihilation operator $\mathbf{A}_{m}^{+}(3.3)$ to the Euler-Lagrange equation in explicit form (2.8) we obtain:

$$
\sum_{l=0}^{k} \mathbf{A}_{m}^{+}\left[\frac{\partial L_{n-l}}{\partial x_{n}}\left(x_{n+k-l}, x_{n+k-1-l}, \ldots, x_{n-l}\right)\right]=0 .
$$

Using the result of Lemma (3.1), as $x_{n+k}$ must be evaluated along the solutions of equation (2.1), and the definition of the annihilation operators (3.3) we have the 
following result:

$\mathbf{A}_{m}^{+}\left[\frac{\partial L_{n-l}}{\partial x_{n}}\left(x_{n+k-l}, \ldots, x_{n-l}\right)\right]= \begin{cases}0 & \text { if } l<m, \\ -\frac{\partial F_{n}}{\partial x_{n-k}} \frac{\partial^{2} L_{n-l}}{\partial x_{n} \partial x_{n-m}}\left(x_{n+k-l}, \ldots, x_{n-l}\right) & \text { if } m \leq l \leq k-1 \\ \mathbf{A}_{m}^{+}\left[\frac{\partial L_{n-k}}{\partial x_{n}}\left(x_{n}, \ldots, x_{n-k}\right)\right] & \text { if } l=k .\end{cases}$

Introducing this results in (3.23) we have:

$$
\mathbf{A}_{m}^{+}\left[\frac{\partial L_{n-k}}{\partial x_{n}}\left(x_{n}, \ldots, x_{n-k}\right)\right]=\frac{\partial F_{n}}{\partial x_{n-k}} \sum_{l=m}^{k-1} \frac{\partial^{2} L_{n-l}}{\partial x_{n-k-l} \partial x_{n}}\left(x_{n+k-l}, \ldots, x_{n-l}\right) .
$$

Then dividing (3.25) by $\partial F_{n} / \partial x_{n-k}$ and differentiating with respect to $x_{n-k}$ we get the system (3.21).

Reasoning in the same way with (3.12) and the backward annihilation operators (3.15) we obtain the system (3.22). This concludes the proof.

Remark 3.5. We underline that the system (3.21) consist of at least $k-1$ equations. In fact each equation in the system (3.21) can be complemented by some additional conditions given by the fact that $F$ in (2.1) depends on the variables $x_{n+i}$ for $i=-k, \ldots, k-1$, while $L_{n-k}=L_{n-k}\left(x_{n}, \ldots, x_{n-k}\right)$. Therefore, we must require to each equation in (3.21) to be independent of additional variables $x_{n+k-r}, r=$ $1, \ldots, k-1$. This implies that the equations

$$
\frac{\partial^{2}}{\partial x_{n-k} \partial x_{n+k-r}}\left\{\left(\frac{\partial F_{n}}{\partial x_{n-k}}\right)^{-1} \mathbf{A}_{m}^{+}\left[\frac{\partial L_{n-k}}{\partial x_{n}}\left(x_{n}, \ldots, x_{n-k}\right)\right]\right\}=0,
$$

for $m, r=1, \ldots, k-1$ must hold. In the simpler, yet usual, case when $F_{n}$ in (2.1) is a rational function of its argument the $k-1$ conditions in (3.26) can be replaced by taking the numerator and then the coefficients with respect to to the variables $x_{n+k-r}$ for $r=1, \ldots, k-1$ in equation (3.21).

Remark 3.6. We remark that the result of Theorem (3.4) do not require to the recurrence relation (2.1) to be defined in both directions, nor it requires to it to be rational. See Section 4 for examples of non-rational recurrence relations admitting Lagrangians.

Theorem 3.4 is an effective tool to compute Lagrangians or to prove if a given difference equation of even order do not possess one. In fact we can state the following:

Corollary 3.5. Assume that the general solution of the system of linear partial differential equation (3.21) (or (3.22) ) associated with a given forward difference equation (2.1) (or backward difference equation (3.12)), with $k>1$, gives raise to a trivial Lagrangian. Then there exists no non-trivial Lagrangian for the forward difference equation (2.1) (or for the backward difference equation (3.12)).

Proof. Assume by contradiction that equation (2.1) possess a non-trivial Lagrangian $L_{n}$. From Theorem 3.4 such Lagrangian will be a particular solution of the system (3.21). However, since the general solution of the linear system (3.21) is trivial also $L_{n}$ must be trivial. This is a contradiction. Reasoning in the same way with 
(3.12) and the system (3.22) we obtain another contradiction. This concludes the proof.

To practically the problem of finding a discrete Lagrangian $L=L_{n}$ we propose the following algorithmic steps.

(1) Introduce the function:

$$
\ell_{n-k}\left(x_{n}, \ldots, x_{n-k}\right)=\frac{\partial L_{n-k}}{\partial x_{n}}\left(x_{n}, \ldots, x_{n-k}\right) .
$$

(2) Then the system (3.21) becomes:

$$
\frac{\partial}{\partial x_{n-k}}\left\{\left(\frac{\partial F_{n}}{\partial x_{n-k}}\right)^{-1} \mathbf{A}_{m}^{+}\left[\ell_{n-k}\left(x_{n}, \ldots, x_{n-k}\right)\right]\right\}=0,
$$

where $m=1, \ldots, k-1$. That is the system (3.21) reduces to a linear system of second-order partial differential equations for $\ell_{n-k}$.

(3) The next step depends on the functional form of the the system (3.28):

(a) If the system (3.28) is rational in $x_{n+k-r}, r=1, \ldots, k-1$ take the numerator of each equation and then the coefficients with respect to the variables $x_{n+k-r}, r=1, \ldots, k-1$. This gives the final system of linear partial differential equations to solve.

(b) If the system (3.28) is not rational consider the derivatives of each equation with respect to the variables $x_{n+k-r}, r=1, \ldots, k-1$ :

$$
\frac{\partial^{2}}{\partial x_{n-k} \partial x_{n+k-r}}\left\{\left(\frac{\partial F_{n}}{\partial x_{n-k}}\right)^{-1} \mathbf{A}_{m}^{+}\left[\ell_{n-k}\left(x_{n}, \ldots, x_{n-k}\right)\right]\right\}=0 \text {. }
$$

This gives the final system of linear partial differential equations to solve.

In both cases solve the resulting final system using, e.g. a computer algebra system like Maple or Mathematica.

(4) Recover the Lagrangian is recovered through integration and translation:

$$
L_{n}\left(x_{n+k}, \ldots, x_{n}\right)=\int^{x_{n+k}} \ell_{n}\left(y, x_{n+k-1}, \ldots, x_{n}\right) \mathrm{d} y+\hat{\ell}_{n}\left(x_{n+k-1}, \ldots, x_{n}\right) .
$$

(5) Fix the form of the remaining arbitrary functions using the compatibility conditions given by equation (3.25).

(6) Check if the Euler-Lagrange equation (2.8) is identically satisfied.

If for technical reasons it is better to use the conditions (3.22), we can perform the steps (i-vi) using the following function:

$$
\tilde{\ell}_{n}\left(x_{n+k}, \ldots, x_{n}\right)=\frac{\partial L_{n}}{\partial x_{n}}\left(x_{n+k}, \ldots, x_{n}\right) .
$$

Instead of the system (3.28) we will solve the analogous system of second-order linear partial differential equations for $\widetilde{\ell}_{n}$ obtained from (3.22). Again the Lagrangian will be recovered through the integration of equation (3.31) and the remaining arbitrary functions will be fixed using the Euler-Lagrange themselves as compatibility conditions. However, we underline that, if needed, it is possible to mix the forward and the backward approach in order to simplify the conditions. See subsection 4.2 for an example of such occurrence. 
Remark 3.7. In the case when $k=2$ we have that the systems (3.21) and (3.22) actually consist of a single equations:

$$
\frac{\partial}{\partial x_{n-2}}\left\{\left(\frac{\partial F_{n}}{\partial x_{n-2}}\right)^{-1} \mathbf{A}_{1}^{+}\left[\ell_{n-2}\left(x_{n}, x_{n-1}, x_{n-2}\right)\right]\right\} \text {. }
$$

and

$$
\frac{\partial}{\partial x_{n+2}}\left\{\left(\frac{\partial \widetilde{F}_{n}}{\partial x_{n+2}}\right)^{-1} \mathbf{A}_{1}^{-}\left[\widetilde{\ell}_{n}\left(x_{n+2}, x_{n+1}, x_{n}\right)\right]\right\} .
$$

If $F$ is rational to obtain the final system one needs to consider the coefficients with respect to $x_{n+1}$, otherwise to differentiate with respect to it in (3.32a). The same with respect to $\widetilde{F}$ and $x_{n-1}$ in (3.32b).

In the next section we see some examples of the theory we presented above.

\section{EXAmPLES}

In this section we present several examples, both positive and negative, of the application of the method discussed in the previous one.

We mainly present autonomous examples. In all autonomous examples the conditions presented in Theorem 3.4 hold simply dropping the subscript $n$. In principle, autonomous systems can have non-autonomous Lagrangians. In the continuous case several instances of this fact are known [2,43, 44. All the examples of autonomous difference equations presented in this paper and known to the authors also have an autonomous Lagrangian. Therefore, the problem of the finding a nonautonomous (up to equivalence) discrete Lagrangian generating an autonomous difference equation is, up to our knowledge, open. In the last subsection we present a non-autonomous example, the $\mathrm{d} P_{\mathrm{I}}^{(2)}$ equation which shows how the method works in full generality.

Before going on, we note that in the working of the presented examples we will employ extensively the computational techniques and reasoning we introduced for the first time in the proofs of Lemmas 2.1 and 2.4 .

4.1. The autonomous $\mathrm{d} P_{\mathrm{II}}^{(2)}$ equation. Let us consider the autonomous $\mathrm{d} P_{\mathrm{II}}^{(2)}$ equation [9, 31]:

$$
\begin{aligned}
x_{n+2}\left(1-x_{n+1}^{2}\right)+x_{n-2}\left(1-x_{n-1}^{2}\right) & =\left(x_{n+1}+x_{n-1}\right)\left[x_{n}\left(x_{n+1}+x_{n-1}\right)+C\right] \\
& -\frac{A x_{n}+B}{1-x_{n}^{2}} .
\end{aligned}
$$

We start then from equation (3.32a) which, when $F$ is given by solving (4.1) with respect to $x_{n+2}$, is:

$$
\begin{aligned}
& {\left[\left(2 x_{n-1}+2 x_{n+1}\right) x_{n}+2 x_{n-2} x_{n-1}+C\right] \frac{\partial^{2} \ell}{\partial x_{n-2}^{2}}} \\
& \quad+\left(1-x_{n-1}^{2}\right) \frac{\partial^{2} \ell}{\partial x_{n-1} \partial x_{n-2}}+2 x_{n-1} \frac{\partial \ell}{\partial x_{n-2}}=0,
\end{aligned}
$$

with $\ell=\ell\left(x_{n}, x_{n-1}, x_{n-2}\right)$. Since there is no dependence on $x_{n+1}$ we can take coefficients with respect and, upon solving with respect to higher order derivatives, 
we obtain:

$$
\frac{\partial^{2} \ell}{\partial x_{n-2}^{2}}=0, \quad \frac{\partial^{2} \ell}{\partial x_{n-1} \partial x_{n-2}}=\frac{2 x_{n-1}}{x_{n-1}{ }^{2}-1} \frac{\partial \ell}{\partial x_{n-2}}
$$

The solution of (4.3) is:

$$
\ell\left(x_{n}, x_{n-1}, x_{n-2}\right)=\left(x_{n-1}^{2}-1\right) x_{n-2} \ell_{1}^{\prime}\left(x_{n}\right)+\frac{\partial \ell_{2}}{\partial x_{n}}\left(x_{n}, x_{n-1}\right) .
$$

Applying twice the translation operator in the positive direction and integrating (4.4) using (3.30) we obtain:

$$
\begin{aligned}
L\left(x_{n+2}, x_{n+1}, x_{n}\right) & =\left(x_{n+1}^{2}-1\right) x_{n} \ell_{1}\left(x_{n+2}\right) \\
& +\ell_{2}\left(x_{n+2}, x_{n+1}\right)+\ell_{3}\left(x_{n+1}, x_{n}\right) .
\end{aligned}
$$

Using the arbitrariness of $\ell_{2}\left(x_{n+2}, x_{n+1}\right)$ we replace it by

$$
\ell_{2}\left(x_{n+2}, x_{n+1}\right)-\ell_{3}\left(x_{n+2}, x_{n+1}\right) \text {. }
$$

This allows to get rid of a total derivative in (4.5) and we are left with the simplified Lagrangian:

$$
L\left(x_{n+2}, x_{n+1}, x_{n}\right)=\left(x_{n+1}^{2}-1\right) x_{n} \ell_{1}\left(x_{n+2}\right)+\ell_{2}\left(x_{n+2}, x_{n+1}\right) .
$$

We must now check that the compatibility condition (3.25) is satisfied. Inserting (4.7) in it we obtain:

$$
\frac{\partial^{2} \ell_{2}}{\partial x_{n-1} \partial x_{n}}\left(x_{n}, x_{n-1}\right)+2 x_{n} \ell_{1}\left(x_{n+1}\right)=\left[\left(2 x_{n-1}+2 x_{n+1}\right) x_{n}+C\right] \ell_{1}^{\prime}\left(x_{n}\right) .
$$

Differentiating (4.8) with respect to $x_{n+1}$ we obtain

$$
\ell_{1}^{\prime}\left(x_{n}\right)=\ell_{1}^{\prime}\left(x_{n+1}\right) .
$$

This last equation implies that $\ell_{1}^{\prime}\left(x_{n}\right)=K_{1}$ so that $\ell_{1}\left(x_{1}\right)=K_{1} x_{n}$. Substituting in (4.8) we have:

$$
\frac{\partial^{2} \ell_{2}}{\partial x_{n-1} \partial x_{n}}\left(x_{n}, x_{n-1}\right)=K_{1}\left(2 x_{n-1} x_{n}+C\right) .
$$

The solution of this last PDE is given by:

$$
\ell_{2}\left(x_{n}, x_{n-1}\right)=\frac{K_{1}}{2} x_{n} x_{n-1}\left(x_{n} x_{n-1}+2 C\right)+\ell_{4}\left(x_{n}\right)+\ell_{5}\left(x_{n-1}\right) .
$$

As before we can replace $\ell_{4}\left(x_{n}\right)$ by $\ell_{4}\left(x_{n}\right)-\ell_{5}\left(x_{5}\right)$ and inserting (4.11) and the value of $\ell_{1}$ into (4.7) we obtain:

$L\left(x_{n+2}, x_{n+1}, x_{n}\right)=K_{1}\left[\left(x_{n+1}^{2}-1\right) x_{n} x_{n+2}+\frac{1}{2} x_{n+1} x_{n}\left(x_{n+1} x_{n}+2 C\right)\right]+\ell_{4}\left(x_{n+1}\right)$

where we eliminated the total differences. Now computing the Euler-Lagrange equations corresponding to (4.12) on the solutions of (4.1) we obtain:

$$
\ell_{4}^{\prime}\left(x_{n}\right)=K_{1} \frac{A x_{n}+B}{x_{n}^{2}-1} .
$$

Solving this last equation with respect to $\ell_{4}\left(x_{n}\right)$ we obtain:

$$
\ell_{4}\left(x_{n}\right)=\frac{K_{1}}{2}\left[(A+B) \log \left(x_{n}-1\right)+(A-B) \log \left(x_{n}+1\right)\right]+K_{2} .
$$


The arbitrary constant is inessential to the Lagrangian, so we can safely set $K_{2}=0$. Finally, inserting (4.14) into (4.12) and rescaling we obtain:

$$
\begin{aligned}
L\left(x_{n+2}, x_{n+1}, x_{n}\right) & =\left(x_{n+1}^{2}-1\right) x_{n} x_{n+2}+\frac{1}{2} x_{n+1} x_{n}\left(x_{n+1} x_{n}+2 C\right) \\
& +\frac{1}{2}\left[(A+B) \log \left(x_{n+1}-1\right)+(A-B) \log \left(x_{n+1}+1\right)\right] .
\end{aligned}
$$

This is the Lagrangian for equation (4.1).

4.2. The Q.iii) equation. Consider the following equation introduced in [22]:

$$
\begin{aligned}
& \left(x_{n-2} x_{n-1}^{2}-x_{n+1}^{2} x_{n+2}-C x_{n-1}+C x_{n+1}\right) x_{n} \\
+ & \left(\frac{C}{2} x_{n-2}+B\right) x_{n-1}-\left(\frac{C}{2} x_{n+2}+B\right) x_{n+1}=\frac{\alpha}{\beta} x_{n}\left(x_{n+1}-x_{n-1}\right) .
\end{aligned}
$$

We will show that this equation has no Lagrangian.

We start then from equation (3.32a), which when $x_{n+2}$ is given by Q.iii) is:

$$
\begin{aligned}
& {\left[\left(4 x_{n} x_{n-2} x_{n-1}-2 C x_{n}+C x_{n-2}+2 B\right) \beta+2 \alpha x_{n}\right] \frac{\partial^{2} \ell}{\partial x_{n-2}^{2}} } \\
- & {\left[x_{n-1}\left(2 x_{n} x_{n-1}+C\right) \frac{\partial^{2} \ell}{\partial x_{n-1} \partial x_{n-2}}-\frac{\partial \ell}{\partial x_{n-2}}\left(4 x_{n} x_{n-1}+C\right)\right] \beta=0, }
\end{aligned}
$$

where $\ell=\ell\left(x_{n}, x_{n-1}, x_{n-2}\right)$. It is possible to solve directly equation (4.16), but the solution is quite involved. So instead of starting by solving this equation, we search for another compatibility condition. From equation (3.25) differentiating with respect to $x_{n+1}$ and translating backward once we obtain:

$$
\frac{\partial^{2} \ell}{\partial x_{n-1} \partial x_{n-2}}=0
$$

Solving equations (4.17) and (4.16) together we obtain

$$
\ell\left(x_{n}, x_{n-1}, x_{n-2}\right)=\ell_{1}\left(x_{n}, x_{n-1}\right) .
$$

Using the definition (3.30) we have that the possible Lagrangian for equation (Q.iii) is:

$$
L\left(x_{n+2}, x_{n+1}, x_{n}\right)=\ell_{1}\left(x_{n+2}, x_{n+1}\right)+\ell_{2}\left(x_{n+1}, x_{n}\right) .
$$

This Lagrangian (4.19) is clearly non-normal. As $\partial L / \partial x_{n+1} \partial x_{n} \neq 0$, we have from Lemma 2.4 that Lagrangian (4.19) can define a difference equation of order two at most. This is a contradiction, and we obtain that equation Q.iii) does not possess a Lagrangian.

4.3. Examples from [53]. In [53] the Ostrogradsky transformation was used to find Poisson structures for periodic reductions of arbitrary order of four partial difference equations. These Poisson structure were found exploiting the Lagrangian formulation for those partial difference equations. Here we will show that these Lagrangians can be derived applying Theorem 3.4. Due to the algorithmic nature of the process we will present the details of the derivation in one of the four cases. All the other examples can be carried out in the same way, so we will not discuss them in details.

In [53] the following four Lagrangians of order $p+q$ are presented:

$$
L_{\mathrm{KdV}}=x_{n} x_{n+p}-x_{n} x_{n+q}-\log \left(x_{n}-x_{n+p+q}\right),
$$




$$
\begin{aligned}
L_{\mathrm{plusKdV}} & =x_{n} x_{n+p}+x_{n} x_{n+q}-\log \left(x_{n}+x_{n+p+q}\right), \\
L_{\mathrm{LV}} & =x_{n} x_{n+p}-x_{n} x_{n+q}-F\left(x_{n}-x_{n+p+q}\right), \\
L_{\mathrm{ASdL}} & =x_{n} x_{n+p}+x_{n} x_{n+q}-F\left(x_{n}+x_{n+p+q}\right),
\end{aligned}
$$

where $p, q \in \mathbb{N}$ such that $p<q$ and $\operatorname{gdc}(p, q)=1$ and the function $F(x)$ is defined by the following integral:

$$
F(x)=\int_{0}^{x} \log \left(1+e^{t}\right) \mathrm{d} t .
$$

These Lagrangian take their names from the fact that each of them arises as $(p,-q)$ reduction of the discrete two dimensional Lagrangian for the discrete KdV equation, the plus-KdV equation, the discrete Lotka-Volterra equation [38] and the Adler-Startsev discretization of the Liouville equation [1] respectively. The EulerLagrange equation of these Lagrangians are respectively:

$$
\begin{gathered}
x_{n+p}+x_{n-p}-x_{n+q}-x_{n-q}-\frac{1}{x_{n}-x_{n+p+q}}+\frac{1}{x_{n-p-q}-x_{n}}=0, \\
x_{n+p}+x_{n-p}-x_{n+q}-x_{n-q}-\frac{1}{x_{n}+x_{n+p+q}}-\frac{1}{x_{n-p-q}+x_{n}}=0, \\
x_{n+p}+x_{n-p}-x_{n+q}-x_{n-q}+\log \left(\frac{1+e^{x_{n}-x_{n+q+p}}}{1+e^{x_{n-p-q}-x_{n}}}\right)=0,
\end{gathered}
$$

$$
x_{n+p}+x_{n-p}+x_{n+q}+x_{n-q}+\log \left[\left(1+e^{x_{n}+x_{n+q+p}}\right)\left(1+e^{x_{n-p-q}+x_{n}}\right)\right]=0 .
$$

We are going to prove the following result:

Lemma 4.1. All the Lagrangians (4.20) can be derived using the conditions (3.21) and (3.22) from Theorem 3.4.

Proof. In the case of equation (4.22a) the forward annihilation operators (3.3) have the following expression:

$$
\begin{aligned}
& \mathbf{A}_{m}^{+}=\frac{1}{D_{\mathrm{KdV}}^{2}} \frac{\partial}{\partial x_{n-m}}, \quad m \neq p, q, \\
& \mathbf{A}_{p}^{+}=\frac{1}{D_{\mathrm{KdV}}^{2}}\left[\frac{\partial}{\partial x_{n-p}}+\left(x_{n}-x_{n-p-q}\right)^{2} \frac{\partial}{\partial x_{n-p-q}}\right], \\
& \mathbf{A}_{q}^{+}=\frac{1}{D_{\mathrm{KdV}}^{2}}\left[\frac{\partial}{\partial x_{n-p}}-\left(x_{n}-x_{n-p-q}\right)^{2} \frac{\partial}{\partial x_{n-p-q}}\right],
\end{aligned}
$$

where:

$$
D_{\mathrm{KdV}}=1-\left(x_{n}-x_{n-p-q}\right)\left(x_{n+p}+x_{n-p}-x_{n+q}-x_{n-q}\right) .
$$

Using these operators we have that the differential conditions on the Lagrangian (3.21) are given by:

$$
\frac{\partial^{2} \ell}{\partial x_{n-m} \partial x_{n-p-q}}=0, \quad m \neq p, q
$$




$$
\begin{array}{rlrl}
\left(x_{n}-x_{n-q-p}\right)^{2} \frac{\partial^{2} \ell}{\partial x_{n-q-p}^{2}} & +\frac{\partial^{2} \ell}{\partial x_{n-q-p} \partial x_{n-p}} \\
& +2\left(x_{n-q-p}-x_{n}\right) \frac{\partial \ell}{\partial x_{n-q-p}}=0, & & \\
\left(x_{n}-x_{n-q-p}\right)^{2} \frac{\partial^{2} \ell}{\partial x_{n-q-p}^{2}} & -\frac{\partial^{2} \ell}{\partial x_{n-q-p} \partial x_{n-q}} \\
& +2\left(x_{n-q-p}-x_{n}\right) \frac{\partial \ell}{\partial x_{n-q-p}}=0, &
\end{array}
$$

Now we prove that solving the conditions (4.25) we find the Lagrangian (4.20a). First of all, we note that the equation (4.22a) depends only on the seven points $x_{n}$, $x_{n \pm p}, x_{n \pm q}$ and $x_{n \pm(p+q)}$. Therefore, we can make the simplifying assumption:

$$
L=L\left(x_{n+p+q}, x_{n+q}, x_{n+p}, x_{n}\right) .
$$

With this assumption the first set of equation (4.25a) is identically satisfied. Then the solution of equations (4.25b) and (4.25c) is given by:

$$
\ell=\ell_{2}\left(x_{n-q}, x_{n-p}, x_{n}\right)+\int \frac{\ell_{1}\left(x_{n}, x_{n-q}+\frac{1+\left(x_{n-p-q}-x_{n}\right) x_{n-p}}{x_{n}-x_{n-p-q}}\right)}{\left(x_{n-p-q}-x_{n}\right)^{2}} \mathrm{~d} x_{n-p-q} .
$$

Following the definition (3.27) we obtain that a Lagrangian for equation (4.22a) must have the following form:

(4.28)

$$
\begin{aligned}
L\left(x_{n+p+q}, x_{n+q}, x_{n+p}, x_{n}\right) & =\ell_{2}\left(x_{n+p}, x_{n+q}, x_{n}\right) \\
& +\iint \frac{\ell_{1}\left(x_{n+p+q}, x_{n+p}+\frac{1+\left(x_{n}-x_{n+p+q}\right) x_{n+q}}{x_{n+p+q}-x_{n}}\right)}{\left(x_{n}-x_{n+p+q}\right)^{2}} \mathrm{~d} x_{n} \mathrm{~d} x_{n+p+q} .
\end{aligned}
$$

Here we used the arbitrariness of $\ell_{2}$ to change it to $\partial \ell_{2} / \partial x_{n}$ in order to keep the expression for $L$ as simple as possible. Deriving the Euler-Lagrange equations of the Lagrangian (4.28), applying the operator $\mathbf{A}_{p}^{+}$(4.23b) then differentiating with respect to $x_{n+p}$ we obtain:

$$
\frac{\partial \ell_{1}}{\partial \xi}=0, \quad \xi=x_{n+p}+\frac{1+\left(x_{n}-x_{n+p+q}\right) x_{n+q}}{x_{n+p+q}-x_{n}} .
$$

This greatly simplifies the expression in (4.28) to:

$$
L\left(x_{n+p+q}, x_{n+q}, x_{n+p}, x_{n}\right)=\ell_{2}\left(x_{n+p}, x_{n+q}, x_{n}\right)-\int \frac{\ell_{1}\left(x_{n+p+q}\right)}{x_{n}-x_{n+p+q}} \mathrm{~d} x_{n+p+q} .
$$

Computing the Euler-Lagrange equations of (4.30) then applying the operators $\mathbf{A}_{p}^{+}$ (4.23b) and $\mathbf{A}_{q}^{+}$(4.23c) we obtain that the function $\ell_{2}$ must satisfy the following system of PDEs:

$$
\begin{aligned}
& \frac{\partial^{2} \ell_{2}}{\partial x_{n-p} \partial x_{n}}\left(x_{n-q}, x_{n-p}, x_{n}\right)=-\ell_{1}\left(x_{n}\right), \\
& \frac{\partial^{2} \ell_{2}}{\partial x_{n-q} \partial x_{n}}\left(x_{n-q}, x_{n-p}, x_{n}\right)=\ell_{1}\left(x_{n}\right) .
\end{aligned}
$$


Introducing $L_{1}\left(x_{n}\right)=\int \ell_{1}\left(x_{n}\right) \mathrm{d} x_{n}$ and solving (4.31) we obtain the following form for the Lagrangian of (4.22a):

$$
\begin{aligned}
L\left(x_{n+p+q}, x_{n+q}, x_{n+p}, x_{n}\right) & =\ell_{3}\left(x_{n+p}, x_{n+q}\right)+\left(x_{n+p}-x_{n+q}\right) L_{1}\left(x_{n}\right) \\
& +\ell_{4}\left(x_{n+p+q}\right)+\ell_{5}\left(x_{n+q}\right)-\int \frac{L_{1}^{\prime}\left(x_{n+p+q}\right)}{x_{n}-x_{n+p+q}} \mathrm{~d} x_{n+p+q} .
\end{aligned}
$$

From the arbitrariness of $\ell_{4}\left(x_{n+p+q}\right)$ we can write

$$
\ell_{4}\left(x_{n+p+q}\right)=\widetilde{\ell}_{4}\left(x_{n+p+q}\right)-\ell_{5}\left(x_{n+p+q}\right)
$$

and since

$$
\ell_{5}\left(x_{n+p+q}\right)-\ell_{5}\left(x_{n+p}\right)=\sum_{l=0}^{q}\left[\ell_{5}\left(x_{n+p+l+1}\right)-\ell_{5}\left(x_{n+p+l}\right)\right] \stackrel{\text { t.d. }}{=} 0
$$

we have the following simplification in (4.32):

$$
\begin{aligned}
L\left(x_{n+p+q}, x_{n+q}, x_{n+p}, x_{n}\right) & =\ell_{3}\left(x_{n+p}, x_{n+q}\right)+\left(x_{n+p}-x_{n+q}\right) L_{1}\left(x_{n}\right) \\
& +\ell_{4}\left(x_{n+p+q}\right)-\int \frac{L_{1}^{\prime}\left(x_{n+p+q}\right)}{x_{n}-x_{n+p+q}} \mathrm{~d} x_{n+p+q} .
\end{aligned}
$$

In (4.35) for sake of simplicity we dropped the tilde in $\ell_{4}\left(x_{n+p+q}\right)$. From the Lagrangian (4.35) we obtain the following Euler-Lagrange equation:

$$
\begin{aligned}
& \int \frac{L_{1}^{\prime}\left(x_{n+q+p}\right)}{\left(x_{n}-x_{n+q+p}\right)^{2}} \mathrm{~d} x_{n+q+p}+\frac{\partial \ell_{3}}{\partial x_{n}}\left(x_{n+p-q}, x_{n}\right)-L_{1}\left(x_{n+p}\right) \\
+ & \frac{\partial \ell_{3}}{\partial x_{n}}\left(x_{n}, x_{n+q-p}\right)+L_{1}\left(x_{n+q}\right)+\left(x_{n-q}-x_{n-p}\right) L_{1}^{\prime}\left(x_{n}\right) \\
+ & \ell_{4}^{\prime}\left(x_{n}\right)+\frac{L_{1}^{\prime}\left(x_{n}\right)}{x_{n}-x_{n-q-p}}=0 .
\end{aligned}
$$

Confronting (4.36) with (4.22a) it is natural to make the linear ansatz for $L_{1}\left(x_{n}\right)$, i.e. $L_{1}\left(x_{n}\right)=K x_{n}$ with $K$ a constant 1 . Using this ansatz the Euler-Lagrange equation (4.36) becomes:

$$
\begin{aligned}
& \frac{K}{x_{n}-x_{n+q+p}}+\frac{\partial \ell_{3}}{\partial x_{n}}\left(x_{n+p-q}, x_{n}\right)+\frac{\partial \ell_{3}}{\partial x_{n}}\left(x_{n}, x_{n+q-p}\right) \\
+ & K\left(x_{n+q}+x_{n-q}-x_{n+p}-x_{n-p}\right)+\ell_{4}^{\prime}\left(x_{n}\right)+\frac{K}{x_{n}-x_{n-q-p}}=0 .
\end{aligned}
$$

Since $x_{n+p+q}$ do not depend on $x_{n+p-q}$ and $x_{n+q-p}$ we have that $\partial^{2} \ell_{3} / \partial x_{n+p} \partial x_{n+q}=$ 0 , i.e.

$$
\ell_{3}\left(x_{n+p}, x_{n+q}\right)=\ell_{6}\left(x_{n+p}\right)+\ell_{7}\left(x_{n+q}\right) .
$$

However, exploiting again the arbitrarily of $\ell_{4}\left(x_{n+p+q}\right)$ we can write

$$
\ell_{4}\left(x_{n+p+q}\right)=\tilde{\ell}_{4}\left(x_{n+p+q}\right)-\ell_{6}\left(x_{n+p+q}\right)-\ell_{7}\left(x_{n+p+q}\right) .
$$

\footnotetext{
${ }^{1}$ Alternatively, without making ansatz it is possible to solve 4.22a for $x_{n-p-q}$ and apply the backward annihilation operators 3.15.
} 
Then from a reasoning analogous to the one in formula (4.34) we can remove all the terms in $\ell_{6}$ and in $\ell_{7}$. Therefore we are left with the following Euler-Lagrange equations:

$$
\frac{K}{x_{n}-x_{n+q+p}}+K\left(x_{n+q}+x_{n-q}-x_{n+p}-x_{n-p}\right)+\ell_{4}^{\prime}\left(x_{n}\right)+\frac{K}{x_{n}-x_{n-q-p}}=0 .
$$

Confronting with (4.22a we have $\ell_{4}^{\prime}\left(x_{n}\right)=0$ so that $\ell_{4}\left(x_{n}\right)=$ constant. Since constants are inessentials in Lagrangian we can safely put $\ell_{4}\left(x_{n}\right)=0$. Putting these considerations together we obtained the Lagrangian:

$$
L\left(x_{n+p+q}, x_{n+q}, x_{n+p}, x_{n}\right)=K\left(x_{n+p}-x_{n+q}\right) x_{n}+K \log \left(x_{n}-x_{n+p+q}\right),
$$

which is equivalent to 4.20a if we choose $K=-1$.

In $\mathrm{A}$ we present the conditions (3.21) for the other three equations in (4.22). The proof that the also the other three Lagrangians in (4.20) can be obtained solving these conditions reported in $\mathrm{A}$ runs in the same way and therefore it is omitted.

4.4. The $\mathrm{d} P_{\mathbf{I}}^{(2)}$ equation. In this subsection we consider the following non-autonomous equation:

$$
\begin{aligned}
& x_{n}\left(x_{n+1} x_{n+2}+x_{n-1} x_{n-2}\right)+x_{n} x_{n-1} x_{n+1} \\
+ & 2 x_{n}^{2}\left(x_{n+1}+x_{n+1}\right)+x_{n}\left(x_{n+1}^{2}+x_{n}^{2}+x_{n-1}^{2}\right) \\
+ & c_{3} x_{n}\left(x_{n-1}+x_{n}+x_{n+1}\right)+c_{2} x_{n}=c_{1}+c_{0}(-1)^{n}-n .
\end{aligned}
$$

This equation is the second member of the Painlevé I hierarchy, in short $\mathrm{d} P_{\mathrm{I}}^{(2)}$, and was first derived in 9]. We will now present a derivation of a discrete Lagrangian for such equation.

First, we consider equation (3.32a), which when $F_{n}$ is given by solving (4.42) with respect to $x_{n+2}$ is the following:

$$
\begin{aligned}
{\left[2\left(x_{n}+x_{n-1}\right)\right.} & \left.+x_{n+1}+c_{3}+x_{n-2}\right] \frac{\partial^{2} \ell_{n-2}}{\partial x_{n-2}^{2}} \\
& -x_{n-1} \frac{\partial^{2} \ell}{\partial x_{n-1} \partial x_{n-2}}+\frac{\partial \ell_{n-2}}{\partial x_{n-2}}=0 .
\end{aligned}
$$

Taking the coefficients with respect to $x_{n+1}$ in (4.43) and solving the resulting system of partial differential equations we obtain that $\ell_{n-2}$ has the following form:

$$
\ell_{n-2}=x_{n-2} x_{n-1} \ell_{1, n-2}\left(x_{n}\right)+\ell_{2, n-2}\left(x_{n}, x_{n-1}\right) \text {. }
$$

Using the definition (3.27) and exploiting the arbitrariness of $\ell_{2, n-2}$ we obtain the following expression for a possible Lagrangian of equation (4.42):

$$
L_{n}\left(x_{n+2}, x_{n+1}, x_{n}\right)=x_{n} x_{n+1} \ell_{1, n}\left(x_{n+2}\right)+\ell_{2, n}\left(x_{n+2}, x_{n+1}\right) .
$$

Computing the Euler-Lagrange equations of (4.45) then applying the operator $\mathbf{A}_{1}^{+}$ we obtain the following compatibility condition:

$$
\frac{\partial^{2} \ell_{2, n-2}}{\partial x_{n-1} \partial x_{n}}\left(x_{n}, x_{n-1}\right)=\left(c_{3}+2 x_{n}+2 x_{n-1}+x_{n+1}\right) \ell_{1, n-2}^{\prime}\left(x_{n}\right)-\ell_{1, n-1}\left(x_{n+1}\right)
$$

Differentiating (4.46) with respect to $x_{n+1}$ we obtain:

$$
\ell_{1, n-1}^{\prime}\left(x_{n+1}\right)=\ell_{1, n-2}^{\prime}\left(x_{n}\right) \text {. }
$$


We have that the functional equation (4.47) implies $\ell_{1, n-2}\left(x_{n}\right)=K_{n-2} x_{n}$ where $K_{n-2}$ is a function depending explicitly on $n$. Inserting such value of $\ell_{1, n-2}\left(x_{n}\right)$ into (4.47) we have $K_{n-1}=K_{n-2}$, i.e. $K_{n}=K$ a constant for all $n \in \mathbb{Z}$. So in the end we have $\ell_{1, n-2}\left(x_{n}\right)=K x_{n}$. Substituting this value of $\ell_{1, n-2}\left(x_{n}\right)$ in equation (4.46) we and solving the obtained partial difference equation with respect to $\ell_{2, n-2}$ we have:

$$
\ell_{2, n-2}\left(x_{n}, x_{n-1}\right)=K x_{n} x_{n-1}\left(c_{3}+x_{n}+x_{n-1}\right)+\ell_{3, n-2}\left(x_{n}\right)+\ell_{4, n-2}\left(x_{n-1}\right) .
$$

Now due to the arbitrariness of $\ell_{3, n-2}\left(x_{n}\right)$ we can write

$$
\ell_{3, n-2}\left(x_{n}\right)=\tilde{\ell}_{3, n-2}\left(x_{n}\right)-\ell_{4, n-1}\left(x_{n}\right)
$$

and, since $\ell_{4, n-1}\left(x_{n}\right)-\ell_{4, n-2}\left(x_{n-1}\right) \stackrel{\text { t.d. }}{\equiv} 0$, we obtain the following Lagrangian:

$L_{n}\left(x_{n+2}, x_{n+1}, x_{n}\right)=K x_{n} x_{n+1} x_{n+2}+K x_{n+2} x_{n+1}\left(c_{3}+x_{n+2}+x_{n+1}\right)+\ell_{3, n}\left(x_{n+2}\right)$,

where for sake of simplicity we dropped the tilde in $\ell_{3, n}$. Computing the EulerLagrange equations of (4.50) and substituting the value of $x_{n+2}$ from (4.42) we are left with the following condition:

$$
x_{n} \ell_{3, n-2}^{\prime}\left(x_{n}\right)=K\left[c_{3} x_{n}^{2}+x_{n}^{3}-c_{0}(-1)^{n}+c_{2} x_{n}+n-c_{1}\right] .
$$

Integrating equation (4.51) and rescaling to eliminate the inessential constant $K$ we obtain the following Lagrangian for equation (4.42):

$$
\begin{aligned}
L_{n}\left(x_{n+2}, x_{n+1}, x_{n}\right) & =x_{n} x_{n+1} x_{n+2}+x_{n+2} x_{n+1}\left(c_{3}+x_{n+2}+x_{n+1}\right) \\
& +\frac{x_{n+2}^{3}}{3}+\frac{c_{3} x_{n+2}^{2}}{2}+c_{2} x_{n+2} \\
& +\left[n+2-c_{0}(-1)^{n}-c_{1}\right] \log x_{n+2} .
\end{aligned}
$$

This ends the proof.

\section{Conclusions}

In this paper we discussed the conditions for the existence of a discrete Lagrangian in the case of scalar difference equations of arbitrary even order $2 k$ with $k>1$. Our main result is contained in Theorem 3.4 and Corollary 3.5 which gives a way of computing such discrete Lagrangians.

The usefulness of the method presented in this paper extends also to differential equations. Indeed, from a difference equation of order $2 k$ through the so-called continuum limit procedure we can always obtain a differential equation of order lesser or equal than $2 k$. Applying the same continuum limit to the discrete Lagrangian of such difference equation we obtain a continuous Lagrangian for the corresponding differential equation. To give an example of this occurrences, let us consider the autonomous $\mathrm{d} P_{\mathrm{II}}^{(2)}$ equation (4.1). It was proved in [9] that the autonomous $\mathrm{d} P_{\mathrm{II}}^{(2)}$ equation (4.1) under the following scaling

$$
\begin{gathered}
x_{n}=h u(t), \quad t=n h, \\
A=6+2 \alpha h^{2}+\delta h^{4}, B=\beta h^{5}, C=4+\alpha h^{2},
\end{gathered}
$$

in the limit $h \rightarrow 0$ reduces to the following differential equation:

$$
u^{\text {iv }}-\alpha u^{\prime \prime}-10 u^{2} u^{\prime \prime}+\left[\delta-10\left(u^{\prime}\right)^{2}\right] u+6 u^{5}+2 \alpha u^{3}+\beta=0 .
$$


This equation is a translated version of the fourth-order member of the $P_{\mathrm{II}}$ hierarchy, the $P_{\mathrm{II}}^{(2)}$ equation, introduced in [14]. Applying the scaling (5.1) to the discrete Lagrangian (4.15) in the limit $h \rightarrow 0$ we obtain:

$$
\begin{aligned}
\frac{L}{h^{6}} & =\frac{1}{2}\left(u^{\prime \prime}\right)^{2}-\frac{1}{2} u\left(u^{2}-\alpha\right) u^{\prime \prime}+\left(\alpha+\frac{7}{2} u^{2}\right)\left(u^{\prime}\right)^{2} \\
& +\frac{1}{2} u\left(2 u^{5}+u^{3} \alpha+\delta u+2 \beta\right)+O(h),
\end{aligned}
$$

up to the addition of a total derivative. The leading order term in (5.3) is a Lagrangian for the $P_{\mathrm{II}}^{(2)}$ equation (5.2). As the $P_{\mathrm{II}}^{(2)}$ equation (5.2) is a fourth-order equations we obtain that the Lagrangian in (5.3) is then its unique Lagrangian [13].

On the other hand the converse procedure, i.e. going from continuous Lagrangian equations to discrete ones, is not possible in general. This happens because, in general, discretising a differential equation does not preserves its variational structure. The non-trivial problem of finding discretisation with variational structure is part of the so-called geometric integration theory [5, 6, 34]. We believe that the method presented in this paper can be helpful in the framework of the geometric integration theory. Indeed, the algorithmic test presented in this paper can be used to isolate Lagrangian difference equations out of families of difference equations with the same continuum limit.

The case $k=1$ seems to be more involved and less algorithmic due to the result of Lemma 3.3 It is possible that this reflects the well-known fact that second-order differential equations

$$
u^{\prime \prime}=F\left(t, u, u^{\prime}\right),
$$

admit infinitely many Lagrangians. These Lagrangians $L=L\left(t, u, u^{\prime}\right)$ are generated by the Jacobi Last Multiplier solving the trivial partial differential equation:

$$
\frac{\partial^{2} L}{\partial u^{\prime 2}}=M
$$

where $M$ is a Jacobi Last Multiplier of the second-order differential equation (5.4) 27 29, 45 47. In 37 the Jacobi Last Multiplier was defined for discrete equation, but no relationship with the discrete Lagrangian was given. Further investigations to establish the existence of a formula analogous to (5.5) are needed.

Let us now comments the relationship of our solution of the inverse problem of the discrete calculus of variation with the one given in [26]. As we noticed in the Introduction in [26] an analogous of the homotopy formula for discrete equations was introduced. The homotopy formula presented in [26 can be used to construct a discrete Lagrangian for scalar difference equations (2.1) for all $k \in \mathbb{N}$ as well as for systems of difference equations, and even in the case of partial difference equations. However, there are some restrictions on the applicability of the homotopy formula presented in [26].

To keep the discussion simple we give a comparison between the applicability of the method presented in [26] and our method. First of all, let us state the results of [26] in our setting. Let $P=P\left[x_{n}\right]$ be a difference function, i.e. a function depending on $x_{n}$ and its shifts up to some order $r$. We denote the space of such functions by $\mathcal{A}^{r}$. The Fréchet derivative of $P$ is an operator $\mathbf{D}_{P}: \mathcal{A}^{q} \rightarrow \mathcal{A}^{r}$ defined 
by:

$$
\mathbf{D}_{P}(Q)=\lim _{\varepsilon \rightarrow 0} \frac{P\left[x_{n}+\varepsilon Q\left[x_{n}\right]\right]-P\left[x_{n}\right]}{\varepsilon}
$$

where $Q=Q\left[x_{n}\right]$ is an arbitrary element of $\mathcal{A}^{q}$. Following [26] we have that if the operator $\mathbf{D}_{P}$ is self-adjoint with respect to the $\ell^{2}(\mathbb{Z})$ norm then the difference equation of order $r$ defined by

$$
P\left[x_{n}\right]=0, \quad P \in \mathcal{A}^{r}
$$

is Lagrangian. To prove that the Fréchet derivative defined by a given difference equation $P \in \mathcal{A}^{r}$ is or not self-adjoint is an algorithmic task which can be always accomplished with a finite number of steps. However, a negative answer to this procedure is not definitive. Indeed, even though the operator $\mathbf{D}_{P}$ is not self-adjoint a difference function $\mu \in \mathcal{A}^{r-1}$ such that $\mathbf{D}_{\mu \cdot P}$ is self-adjoint might exist. Now since $\mu\left[x_{n}\right] \in \mathcal{A}^{r-1}$ we have that the difference equation defined by:

$$
\mu\left[x_{n}\right] P\left[x_{n}\right]=0,
$$

is equivalent to equation (5.7). The difference function $\mu\left[x_{n}\right]$ is called a multiplier.

Therefore, to disprove the existence of a Lagrangian for the difference equation (5.7) we must be able to prove that the operator $\mathbf{D}_{\mu \cdot P}$ is not self-adjoint for every multiplier $\mu\left[x_{n}\right]$.

On the other hand, we notice that with our method, multipliers are unessential. Indeed, the annihilation operators (3.3) and (3.15) are uniquely defined by the solutions of the difference equations (5.7) with respect to the highest or the lowest shift of $x_{n}$, namely equations (2.11) and (3.12). Therefore, the conditions stated in Theorem 3.4 are independent of the value of any possible multiplier $\mu \in \mathcal{A}^{r-1}$, since equations (2.1) and (3.12) are unchanged upon multiplication. So, the outcome of the method presented in Section 3 is definitive up to point transformations in the variable $x_{n}$.

Moreover, in an upcoming paper we will consider the problem of classification of variational difference equations of a given order with some mild additional assumption on the functional form of the difference equation (e.g. reversibility). In that contest the conditions of Theorem 3.4 will act as constraint on the functional form of the function $F$ in (2.1). We remark that this problem is non-trivial since using the systems (3.21) and (3.22) as a classification tool requires to derive and solve systems of nonlinear partial difference equations.

Finally, work is in progress to extend the method of the annihilation operators to more general discrete variational problems, like systems of difference equations.

\section{ACKNOWLEDGEMENTS}

We thank Prof. Nalini Joshi, Prof. Maria Clara Nucci and Dr. Dinh Tran for the interesting and fruitful discussion during the preparation of this paper. Moreover, we thank Dr. Dinh T. Tran for pointing out the examples contained in Subsection 4.3. Finally, we express our gratitude to the anonymous referees whose comments and suggestions led to a great improvement of the paper.

GG is supported by the Australian Research Council through Nalini Joshi's Australian Laureate Fellowship grant FL120100094. 


\section{Appendix A. Conditions on the EXistence of A LAgrangian for EQUATIONS 4.22)}

To end the proof of Lemma 4.1 we must show that the Lagrangians (4.20) arise from the conditions (3.21). As the computations are analogous to those presented in Subsection 4.3 we will limit ourselves to present the form of the forward annihilation operators (3.3) and of the corresponding conditions on the existence of a Lagrangian (3.21). The interested reader can check using the ansatz (4.26) that these conditions yield the desired Lagrangians.

In Subsection 4.3 we already discussed the Lagrangian (4.20a), so we start from the Lagrangian (4.20b) and its Euler-Lagrange equations (4.22b). In the case of equation (4.22b) the forward annihilation operators (3.3) have the following expression:

$$
\begin{aligned}
& \mathbf{A}_{m}^{+}=\frac{1}{D_{\text {plusKdV }}^{2}} \frac{\partial}{\partial x_{n-m}}, \quad m \neq p, q, \\
& \mathbf{A}_{p}^{+}=\frac{1}{D_{\text {plusKdV }}^{2}}\left[\frac{\partial}{\partial x_{n-p}}-\left(x_{n}+x_{n-p-q}\right)^{2} \frac{\partial}{\partial x_{n-p-q}}\right], \\
& \mathbf{A}_{q}^{+}=\frac{1}{D_{\text {plusKdV }}^{2}}\left[\frac{\partial}{\partial x_{n-p}}-\left(x_{n}+x_{n-p-q}\right)^{2} \frac{\partial}{\partial x_{n-p-q}}\right],
\end{aligned}
$$

where:

$$
D_{\text {plusKdV }}=1-\left(x_{n}+x_{n-p-q}\right)\left(x_{n+p}+x_{n-p}+x_{n+q}+x_{n-q}\right) .
$$

Using these operators we have that the differential conditions on the Lagrangian (3.21) are given by:

$$
\begin{aligned}
& \frac{\partial^{2} \ell}{\partial x_{n-m} \partial x_{n-p-q}}=0, \quad m \neq p, q, \\
& \left(x_{n}+x_{n-q-p}\right)^{2} \frac{\partial^{2} \ell}{\partial x_{n-q-p}^{2}}-\frac{\partial^{2} \ell}{\partial x_{n-q-p} \partial x_{n-p}} \\
& +2\left(x_{n-q-p}+x_{n}\right) \frac{\partial \ell}{\partial x_{n-q-p}}=0, \\
& \left(x_{n}+x_{n-q-p}\right)^{2} \frac{\partial^{2} \ell}{\partial x_{n-q-p}^{2}}-\frac{\partial^{2} \ell}{\partial x_{n-q-p} \partial x_{n-q}} \\
& +2\left(x_{n-q-p}+x_{n}\right) \frac{\partial \ell}{\partial x_{n-q-p}}=0, \\
& m=p,
\end{aligned}
$$

The Lagrangian obtained in 53 arises solving the system (A.3).

In the case of equation (4.22c) the forward annihilation operators (3.3) have the following expression:

$$
\begin{aligned}
& \mathbf{A}_{m}^{+}=D_{\mathrm{LV}} \frac{\partial}{\partial x_{n-m}}, \quad m \neq p, q, \\
& \mathbf{A}_{p}^{+}=D_{\mathrm{LV}}\left[\frac{\partial}{\partial x_{n-p}}+\left(1+e^{x_{n}-x_{n-p-q}}\right) \frac{\partial}{\partial x_{n-p-q}}\right], \\
& \mathbf{A}_{q}^{+}=D_{\mathrm{LV}}\left[\frac{\partial}{\partial x_{n-p}}-\left(1+e^{x_{n}-x_{n-p-q}}\right) \frac{\partial}{\partial x_{n-p-q}}\right],
\end{aligned}
$$


where:

$$
D_{\mathrm{LV}}=\frac{e^{x_{n+q}+x_{n-q}-x_{n+p}-x_{n-p}+x_{n-p-q}-x_{n}}}{e^{x_{n+q}+x_{n-q}-x_{n+p}-x_{n-p}}\left(1+e^{+x_{n-p-q}-x_{n}}\right)-1} .
$$

Using these operators we have that the differential conditions on the Lagrangian (3.21) are given by:

$$
\begin{aligned}
& \frac{\partial^{2} \ell}{\partial x_{n-m} \partial x_{n-p-q}}=0, \quad m \neq p, q, \\
& \left(1+e^{x_{n}-x_{n-p-q}}\right) \frac{\partial^{2} \ell}{\partial x_{n-q-p}^{2}}+\frac{\partial^{2} \ell}{\partial x_{n-q-p} \partial x_{n-p}} \\
& -e^{x_{n}-x_{n-p-q}} \frac{\partial \ell}{\partial x_{n-q-p}}=0, \\
& m=p, \\
& \left(1+e^{x_{n}-x_{n-p-q}}\right) \frac{\partial^{2} \ell}{\partial x_{n-q-p}^{2}}-\frac{\partial^{2} \ell}{\partial x_{n-q-p} \partial x_{n-q}} \\
& -e^{x_{n}-x_{n-p-q}} \frac{\partial \ell}{\partial x_{n-q-p}}=0,
\end{aligned}
$$

The Lagrangian obtained in [53] arises solving the system (A.6).

In the case of equation (4.22d) the forward annihilation operators (3.3) have the following expression:

$$
\begin{aligned}
& \mathbf{A}_{m}^{+}=D_{\mathrm{ASdL}} \frac{\partial}{\partial x_{n-m}}, \quad m \neq p, q \\
& \mathbf{A}_{p}^{+}=D_{\mathrm{ASdL}}\left[\frac{\partial}{\partial x_{n-p}}-\left(1+e^{x_{n}+x_{n-p-q}}\right) \frac{\partial}{\partial x_{n-p-q}}\right], \\
& \mathbf{A}_{q}^{+}=D_{\mathrm{ASdL}}\left[\frac{\partial}{\partial x_{n-p}}-\left(1+e^{x_{n}+x_{n-p-q}}\right) \frac{\partial}{\partial x_{n-p-q}}\right],
\end{aligned}
$$

where:

$$
D_{\mathrm{ASdL}}=\frac{e^{x_{n-p-q}+x_{n}-x_{n+p}-x_{n+q}-x_{n-q}-x_{n-p}}}{\left(1+e^{x_{n-p-q}+x_{n}}\right)\left(1+e^{x_{n-p-q}+x_{n}}-e^{-x_{n+p}-x_{n+q}-x_{n-q}-x_{n-p}}\right)}
$$

Using these operators we have that the differential conditions on the Lagrangian (3.21) are given by:

$$
\begin{aligned}
& \frac{\partial^{2} \ell}{\partial x_{n-m} \partial x_{n-p-q}}=0, \quad m \neq p, q, \\
& \left(1+e^{x_{n}+x_{n-p-q}}\right) \frac{\partial^{2} \ell}{\partial x_{n-q-p}^{2}}-e^{x_{n}+x_{n-p-q}} \frac{\partial^{2} \ell}{\partial x_{n-q-p} \partial x_{n-p}} \quad m=p, \\
& -\frac{\partial \ell}{\partial x_{n-q-p}}=0 \\
& \left(1+e^{x_{n}+x_{n-p-q}}\right) \frac{\partial^{2} \ell}{\partial x_{n-q-p}^{2}}-e^{x_{n}+x_{n-p-q}} \frac{\partial^{2} \ell}{\partial x_{n-q-p} \partial x_{n-q}} \quad m=q . \\
& -\frac{\partial \ell}{\partial x_{n-q-p}}=0
\end{aligned}
$$

The Lagrangian obtained in [53] arises solving the system (A.9). This ends the proof of Lemma 4.1 . 


\section{REFERENCES}

[1] V. E. Adler and S. Ya. Startsev. Discrete analogues of the Liouville equation. Theor. Math. Phys., 121(2):1484-1495, 1999.

[2] I. Anderson and G. Thompson. The inverse problem of the calculus of variations for ordinary differential equations, volume 98 of Memoirs of the American Mathematical Society. 1992.

[3] V. I. Arnol'd. Lectures on Partial Differential Equations. Universitext. Springer-Verlag, Berlin, 2004.

[4] M. Bruschi, O. Ragnisco, P. M. Santini, and G-Z. Tu. Integrable symplectic maps. Physica D, 49(3):273 - 294, 1991.

[5] C. J. Budd and A. Iserles. Geometric integration: numerical solution of differential equations on manifolds. R. Soc. Lond. Philos. Trans. Ser. A, 357(1754):945-956, 1999.

[6] C. J. Budd and M. D. Piggott. Geometric integration and its applications. In F. Cucker, editor, Handbook of Numerical Analysis, Vol. XI., pages 35-139. North-Holland, Amsterdam, 2003.

[7] G. Caviglia. Helmholtz conditions, covariance, and invariance identities. Int. J. Theor. Phys., 24:377-390, 1985.

[8] M. Crampin, W. Sarlet, E. Martínez, G. B. Byrnes, and G. E. Prince. Towards a geometrical understanding of Douglas' solution of the inverse problem of the calculus of variations. Inverse Problems, 10:245-260, 1994.

[9] C. Cresswell and N. Joshi. The discrete first, second and thirty-fourth Painleve hierarchies. J. Phys. A: Math. Gen., 32:655-669, 1999.

[10] B. Dacorogna. Introduction to the calculus of variations. Imperial College Press, London, 3rd edition, 2015.

[11] G. Darboux. Leçons sur la théorie généle de surfaces, volume II. Hermann, Paris, 1915.

[12] J. Douglas. Solution of the inverse problem of the calculus of variations. Trans. Amer. Math. Soc., 50:71-128, 1941.

[13] M. E. Fels. The inverse problem of the calculus of variations for scalar fourth-order ordinary differential equations. Trans. Amer. Math. Soc., 348:5007-5029, 1996.

[14] H. Flaschka and A. C. Newell. Monodromy- and spectrum-preserving deformations I. Commun. Math. Phys., 76:65-116, 1980.

[15] A. R. Forsyth. Calculus of variations. Dover Publications, Inc., New York, 1960.

[16] R. N. Garifullin, E. V. Gudkova, and I. T. Habibullin. Method for searching higher symmetries for quad-graph equations. J. Phys. A: Math. Theor., 44:325202 (16pp), 2011.

[17] R. N. Garifullin and R. I. Yamilov. Generalized symmetry classification of discrete equations of a class depending on twelve parameters. J. Phys. A: Math. Theor., 45:345205 (23pp), 2012.

[18] R. N. Garifullin and R. I. Yamilov. Integrable discrete nonautonomous quad-equations as Bäcklund auto-transformations for known Volterra and Toda type semidiscrete equations. $J$. Phys.: Conf. Ser., 621:012005 (18pp), 2015.

[19] I. M. Gelfand and S. V. Fomin. Calculus of variations. Prentice Hall, Englewood Cliffs, New Jersey, 1963. translated and edited by R. A. Silverman.

[20] H. Goldstein, C. Poole, and J. Safko. Classical Mechanics. Pearson Education, 2002.

[21] G. Gubbiotti, N. Joshi, D. T. Tran, and C-M. Viallet. Complexity and integrability in 4D bi-rational maps with two invariants, 2018. Preprint on arXiv:1808.04942 [nlin.SI].

[22] G. Gubbiotti, N. Joshi, D. T. Tran, and C-M. Viallet. Integrability properties of a class of $4 \mathrm{D}$ bi-rational maps with two invariants, 2019. In preparation.

[23] G. Gubbiotti and R. I. Yamilov. Darboux integrability of trapezoidal $H^{4}$ and $H^{6}$ families of lattice equations I: First integrals. J. Phys. A: Math. Theor., 50:345205 (26pp), 2017.

[24] H. Helmholtz. Über die physikalische bedutung des princips der kleinsten wirkung. J. Reine Angew. Math., 100:137-166, 1887.

[25] M. Henneaux and L. C. Shepley. Lagrangians for spherically symmetric potentials. J. Math. Phys., 23(11):2101-2107, 1982.

[26] P. E. Hydon and E. L. Mansfield. A variational complex for difference equations. Found. Comp. Math., 4:187-217, 2004.

[27] C. G. J. Jacobi. Sul principio dell'ultimo moltiplicatore, e suo uso come nuovo principio generale di meccanica. Giornale Arcadico di Scienze, Lettere ed Arti, 99:129-146, 1844.

[28] C. G. J. Jacobi. Theoria novi multiplicatoris systemati æquationum differentialium vulgarium applicandi. J. Reine Angew. Math., 29:213-279 and 333-376, 1845. 
[29] C. G. J. Jacobi. Über die Darstellung einer Reihe gegebner Werthe durch eine gebrochne rationale Function. J. für Reine Angewandte Math., 30:127-156, 1846.

[30] C. G. J. Jacobi. Vorlesungen über Dynamik. Nebst fünf hinterlassenen Abhandlungen desselben herausgegeben von A. Clebsch. Druck und Verlag von Georg Reimer, Berlin, 1884.

[31] N. Joshi and C-M. Viallet. Rational Maps with Invariant Surfaces. J. Int. Sys., 3:xyy017 (14pp), 2018

[32] J. Jost and X. Li-Jost. Calculus of variations, volume 64 of Cambridge Studies in Advanced Mathematics. Cambridge University Press, Cambridge, 1998.

[33] M. Juráš. The inverse problem of the calculus of variations for sixth- and eighth-order scalar ordinary differential equations. Acta Appl. Math., 66:25-39, 2001.

[34] S. G. Krantz and H. R. Parks. Geometric integration theory. Cornerstones. Birkhäuser, Boston, MA, 2008.

[35] B. A. Kupershmidt. Discrete Lax equations and differential-difference calculus. Number 123 in Astérisque. Société mathématique de France, 1985.

[36] L. D. Landau and E. M. Lifshitz. Mechanics. Course of Theoretical Physics. Elsevier Science, 1982.

[37] D. Levi and M. A. Rodríguez. The Jacobi last multiplier for linear partial difference equations. J. Phys. A Math. Theor., 45(27):275205, 2012.

[38] D. Levi and R. I. Yamilov. The generalized symmetry method for discrete equations. J. Phys. A: Math. Theor., 42:454012 (18pp), 2009.

[39] D. Levi and R. I. Yamilov. Generalized symmetry integrability test for discrete equations on the square lattice. J. Phys. A: Math. Theor., 44:145207 (22pp), 2011.

[40] J. D. Logan. First integrals in the discrete variational calculus. Aeq. Math., 9:210-220, 1973.

[41] M. C. Nucci. Jacobi last multiplier and Lie symmetries: a novel application of an old relationship. J. Nonlinear Math. Phys., 12:284-304, 2005.

[42] M. C. Nucci and A. M. Arthurs. On the inverse problem of calculus of variations for fourthorder equations. Proc. R. Soc. A, page rspa20090618, 2010.

[43] M. C. Nucci and P. G. L. Leach. Lagrangians galore. J. Math. Phys., 48:123510, 2007.

[44] M. C. Nucci and P. G. L. Leach. Jacobi's last multiplier and Lagrangians for multidimensional systems. J. Math. Phys., 49:073517, 2008.

[45] M. C. Nucci and K. M. Tamizhmani. Lagrangians for dissipative nonlinear oscillators: the method of Jacobi Last Multiplier. J. Nonlinear Math. Phys., 17:167-178, 2010.

[46] M. C. Nucci and K. M. Tamizhmani. Using an old method of Jacobi to derive Lagrangians: a nonlinear dynamical system with variable coefficients. Il Nuovo Cimento B, 125:255-269, 2010.

[47] M. C. Nucci and K. M. Tamizhmani. Lagrangians for biological models. J. Nonlinear Math. Phys. 19, 2012.

[48] P. J. Olver. Applications of Lie Groups to Differential Equations. Springer-Verlag, Berlin, 1986.

[49] B. S. Madhava Rao. On the reduction of dynamical equations to the lagrangian form. Proc. Benaras Math. Soc.(NS), 2:53-59, 1940.

[50] H. Sagan. Introduction to the calculus of variations. Dover Publications, Inc., New York, 1992. Corrected reprint of the 1969 original.

[51] W. Sarlet, G. Thompson, and G. E. Prince. The inverse problem of the calculus of variations: the use of geometrical calculus in Douglas's analysis. Trans. Amer. Math. Soc., 354(7):28972919, 2002.

[52] D. J. Saunders. Thirty years of the inverse problem in the calculus of variations. Rep. Math. Phys., 66:43-53, 2010.

[53] D. T. Tran, P. H. van der Kamp, and G. R. W. Quispel. Poisson brackets of mappings obtained as $(q,-p)$ reductions of lattice equations. Regular and Chaotic Dynamics, 21(6):682-696, 2016.

[54] M. M. Vainberg. Variational methods for the study of nonlinear operators. Holden-Day, San Francisco, 1964.

[55] A. P. Veselov. Integrable maps. Russ. Math. Surveys, 46:1-51, 1991.

[56] R. Vitolo. Variational sequences. In D. Krupka and D. Saunders, editors, Handbook of Global Analysis. Elsevier, 2007. Lecture notes on the subject available at http://poincare.unisalento.it/vitolo/vitolo_files/publications/in-books/varseq.pdf.

[57] V. Volterra. Leçons sur les fonctions de ligne. Gauthier-Villars, Paris, 1916. 
[58] E. T. Whittaker. A Treatise on the Analytical Dynamics of Particles and Rigid Bodies. Cambridge University Press, Cambridge, 1999.

School of Mathematics and Statistics F07, The University of Sydney, NSW 2006, Australia

E-mail address: giorgio.gubbiotti@sydney.edu.au 